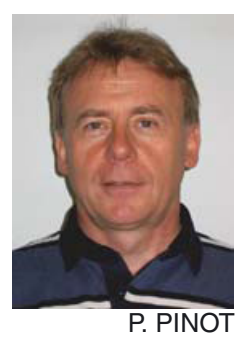

\title{
Détermination de la porosité de roches calcaires : méthode et incertitude de mesure
}

\section{Determining the porosity of limestone: experimental method and uncertainty}

\author{
Patrick PINOT
}

Laboratoire commun de métrologie LNE-LCM/Cnam, CNAM, 61 Rue du Landy, 93210 La Plaine Saint-Denis, France, patrick.pinot@cnam.fr.

Résumé

Une méthode simple inspirée de celle décrite dans la norme internationale NF ISO 5017 est mise en œuvre pour déterminer la porosité ouverte d'échantillons de roche calcaire. Son efficacité est démontrée et les points qui doivent être particulièrement maîtrisés (séchage, imbibition, essuyage) sont mis en évidence en fonction du type de porosité (fine ou large) pour éviter des erreurs grossières. Cette étude montre que la norme NF ISO 5017 établie pour des produits réfractaires façonnés denses est également bien adaptée aux roches solides à structure homogène ayant une porosité totale inférieure à $45 \%$. Cette norme correctement mise en œuvre pour des échantillons de roche permet de déterminer leur porosité ouverte avec une incertitude type composée de l'ordre de $0,1 \%$.

MOTS CLÉS : ROCHE CALCAIRE, POROSITÉ OUVERTE, MÉTHODE GRAVIMÉTRIQUE, INCERTITUDE DE MESURE.

\begin{abstract}
A simple method based on that described in the international standard NF ISO 5017 is implemented to determine the apparent porosity of samples of limestone. Its effectiveness is demonstrated and the points to be particularly controlled (drying, soaking, wiping) to avoid gross errors are highlighted depending on the type of porosity (thin or wide). This study shows that the standard NF ISO 5017 established for dense shaped refractory products is also well suited for solid rock with homogeneous structure having a true porosity of less than $45 \%$. This standard properly implemented for rock samples allows one to determine their apparent porosity with a standard uncertainty of about $0.1 \%$.
\end{abstract}

KEY WORDS: LIMESTONE, APPARENT POROSITY, GRAVIMETRIC METHOD, UNCERTAINTY IN MEASUREMENT.

\section{Introduction}

La plupart des matériaux solides naturels ou artificiels sont poreux et leurs propriétés physiques et chimiques dépendent de la structure et de la taille de leurs pores. En effet, la porosité d'un matériau solide influence sa réactivité chimique et son interaction physique avec les gaz et les liquides. C'est pourquoi le contrôle et la détermination de leur porosité revêt une grande importance industrielle du fait des nombreuses applications très spécifiques de ce paramètre dans divers domaines industriels. Parmi cellesci, on peut citer, par exemple, la conception et le développement de catalyseurs, d'adsorbants industriels, de membranes filtrantes, de céramiques, le stockage de gaz tel que le dihydrogène...

En raison de la complexité et de la diversité des matériaux poreux et de la spécificité de leurs utilisations, il existe de nombreuses techniques pour les caractériser [1]. On en décrit quelques-unes après avoir précisé les notions de base permettant de définir la porosité.

Parmi les matériaux solides naturels, les roches sont bien connues pour être une source de matières premières [2] dans divers domaines tels l'énergie (anthracite...), la métallurgie (bauxite...), la chimie (craie...) ou la construction (granit...). Dans ces exemples, leur utilisation nécessite une extraction sur des sites de gisement. Les roches du sous-sol présentent également un autre intérêt économique et stratégique in situ de très grande importance. En effet, certaines roches poreuses constituent de véritables réservoirs chargés naturellement ou artificiellement d'un fluide tel que l'eau, le pétrole ou 
le gaz naturel $[3,4]$. La compréhension des caractéristiques de ces réservoirs naturels est cruciale. De ce point de vue, les deux propriétés fondamentales qui qualifient des roches sont la porosité et la perméabilité. Pour se rendre compte des enjeux que recouvre ce type de gisement, il suffit de se reporter aux débats que suscitent l'exploitation du gaz de schiste (gaz contenu dans les pores d'une roche rendue imperméable par l'argile) ou le stockage géologique de dioxyde de carbone.

L'objectif de l'étude décrite dans cet article est de mettre en œuvre une méthode inspirée de normes existantes pour la détermination de la porosité de roches par un opérateur non spécialiste de ce domaine, d'analyser le processus métrologique et de démontrer l'efficacité de cette méthode. Pour ce faire, on a choisi de travailler sur des échantillons de roche calcaire. Cette étude a été réalisée avec des moyens simples disponibles au laboratoire; les dispositifs de mesure et les modes opératoires ne sont donc pas optimisés. La méthode employée est fondée sur celle décrite dans une norme internationale qui est commentée. Ensuite, la procédure, les moyens mis en œuvre au laboratoire et les résultats de mesure obtenus sont présentés. Enfin, une analyse du processus de mesure permet de justifier l'incertitude que l'on peut raisonnablement attribuer aux résultats obtenus par cette méthode de mesure.

\section{Généralités}

\subsection{Définitions}

Avant de décrire la méthode utilisée pour déterminer la porosité d'échantillons de roche, il est nécessaire de définir certaines notions indispensables à la compréhension de la spécificité de cette détermination $[1,5]$. Les deux caractéristiques interdépendantes des roches sont la porosité et la perméabilité. Alors que la porosité correspond au volume relatif des espaces vides présents dans le matériau, la perméabilité mesure l'aptitude du matériau à se laisser traverser par un fluide. D'une manière générale, un solide poreux est un solide qui présente des pores, c'est-à-dire des cavités, des canaux ou interstices (plus profonds que larges). Voici quelques définitions.

\subsubsection{Roche}

En géologie, les roches sont des matériaux solides ou liquides constitutifs de la croûte terrestre (ou de celle des planètes). Elles sont composées de minéraux, c'est-à-dire de substances principalement inorganiques et, plus rarement, organiques formées naturellement. On ne considérera ici que des roches solides du type agglomérat, c'est-à-dire des matériaux consolidés dont les dimensions dépassent de plusieurs ordres de grandeur celles des pores. A contrario, les roches solides non consolidées du type agrégat sont constituées de particules individuelles comme par exemple le sable.

\subsubsection{Calcaire}

Les calcaires sont des roches sédimentaires, contenant au moins $50 \%$ de carbonate de calcium, qui ont été formées en milieu subaquatique. Elles peuvent être d'origine organique, biochimique ou détritique. Leurs caractéristiques sont très variables suivant l'âge de la roche, leur milieu de dépôt et dépendent des transformations subies au cours de la diagénèse (processus physico-chimiques et biochimiques de solidification) ou du métamorphisme (transformation par augmentation de la température et/ou de la pression). Par exemple, les marbres sont des calcaires recristallisés sous l'action du métamorphisme. De plus, la présence d'impuretés peut engendrer des modifications des caractéristiques chimiques ou physiques de la roche (couleur, dureté... ).

\subsubsection{Pore}

On distingue plusieurs types de pores en fonction soit de leur structure ou bien de leur forme. On se limite ici à deux types de pores (notions très relatives) : les pores ouverts ou connectés et les pores fermés ou occlus. Les pores ouverts sont ceux qui communiquent avec l'atmosphère, soit directement, soit de proche en proche, et qui peuvent être pénétrés naturellement par un fluide (gaz ou liquide). Les pores fermés sont ceux qui ne peuvent pas être pénétrés par un fluide. De plus, on peut distinguer deux sous-classes de pores ouverts, les pores «borgnes » ouverts à l'atmosphère que par une seule de leurs extrémités et les pores «traversant» ouverts à l'atmosphère à leurs deux extrémités directement ou communiquant avec leurs voisins. Les premiers peuvent adsorber des fluides (gaz ou liquide), mais ne permettent pas un écoulement des fluides à travers le matériau. Les seconds donnent une propriété de perméabilité naturelle à la roche permettant, par exemple, la percolation par gravité.

Il faut préciser que les pores fermés comme les pores ouverts ont une influence sur la masse volumique, la résistance mécanique et la conductivité thermique du matériau, mais, contrairement aux pores ouverts, les pores fermés n'ont aucune influence sur l'écoulement de fluide à travers le matériau et sur l'adsorption de fluide dans le matériau.

\subsubsection{Volume [5]}

On distingue le volume réel du matériau solide poreux comme étant le volume de la partie solide et son volume apparent comme étant la somme des volumes de la matière solide, des pores ouverts et des pores fermés.

\subsubsection{Masse volumique [5]}

La distinction des volumes réel et apparent conduit à définir, d'une part, la masse volumique absolue du matériau solide poreux comme étant le rapport de la masse de sa matière sèche à son volume réel et, d'autre part, sa masse volumique apparente comme étant le rapport de la masse de sa matière sèche à son volume apparent. 


\subsection{Techniques de mesure de la porosité}

Il existe de très nombreuses méthodes de caractérisation de la porosité d'un matériau solide [1]. Chacune d'entre elles est plus ou moins bien adaptée à une texture de matériau, ou à une dimension d'échantillon ou encore à une taille de pores... De plus, chaque méthode ne fournit qu'une caractérisation partielle de la porosité en termes de géométrie des pores ou de capacité de stockage ou d'adsorption (diamètre, surface, volume, répartition...). Le choix de la technique se fait également en fonction de l'application à laquelle est destiné le matériau. Voici quelques exemples permettant d'illustrer la variété des techniques employées.

\subsubsection{Stéréologie [6-8]}

Elle consiste à observer au moyen d'un microscope optique ou électronique une coupe du matériau dont l'image peut être traitée pour augmenter le contraste entre les pores et la matrice. Cette méthode permet d'obtenir certaines caractéristiques géométriques de la porosité comme, entre autres, le rapport de la surface des pores à celle de la matrice pour une image (surface) de la coupe considérée.

\subsubsection{Diffusion de rayonnement [9]}

Cette technique n'est applicable que sur des solides dont les pores et la matrice présentent des caractéristiques (distribution de la taille et de la répartition des pores...) accessibles à partir de la mesure de la distribution angulaire de l'intensité du rayonnement diffusé. La surface du matériau est placée sous un faisceau incident d'un rayonnement électromagnétique monochromatique (rayon lumineux ou rayon $\mathrm{X}$ ) ou de neutrons. Cette technique est particulièrement adaptée pour les pores de dimension moléculaire. Mais l'effet de la rugosité de surface, entre autres, doit être pris en compte, puisqu'elle peut avoir une influence sur la diffusion du rayonnement.

\subsubsection{Pycnométrie [10]}

Elle permet de déterminer la masse volumique apparente à partir de la pénétration d'un fluide, par exemple d'un liquide, dans les pores ouverts. Le choix du liquide est primordial pour la détermination de la porosité. Si le liquide «mouille» mal le solide, il ne pourra pas pénétrer complètement dans les pores et s'il «mouille» très bien le solide, il aura tendance à pénétrer dans tous les pores accessibles à ses molécules. Dans ce cas, on peut s'attendre à une augmentation de la porosité ouverte quand la taille de la molécule sonde diminue. Cette méthode suppose que la masse volumique du liquide considéré reste constante jusqu'à la surface du solide, c'est-à-dire que l'effet d'adsorption du liquide demeure négligeable. De plus, il faut utiliser un liquide parfaitement dégazé, qui soit compatible avec le solide et, en particulier, qu'il ne provoque pas un gonflement ou une désagrégation du matériau. Dans certains cas, il est souvent préférable d'utiliser un gaz plutôt qu'un liquide $[11,12]$. Par exemple avec de l'hélium, on peut mesurer la masse volumique absolue du matériau. Ainsi pour les roches, la méthode repose sur les mesures séparées de la masse sèche de poudre de roche par pesée et celle de son volume au moyen d'un pycnomètre à hélium.

\subsubsection{Adsorption de gaz [13, 14]}

Cette méthode est utilisée pour caractériser la capacité de physisorption de matériaux poreux (zéolites, catalyseurs, adsorbants industriels...). Il existe plusieurs techniques de mesure de quantité adsorbée. Par exemple, on met en œuvre des méthodes volumétriques pour déterminer les isothermes en utilisant de l'azote ou bien du krypton à basse température ou des méthodes gravimétriques pour l'adsorption de vapeur à température ambiante. On peut également déterminer l' aire de la surface accessible à l'adsorption en appliquant une méthode BET (Brunauer-Emmett-Teller) qui permet de calculer l'aire à partir d'isothermes de physisorption. Il existe plusieurs causes d'erreur qui proviennent, par exemple, des interactions adsorbant/adsorbat et du fait que la théorie BET repose uniquement sur l'extension du mécanisme de Langmuir à l'adsorption multicouche.

\subsubsection{Autres méthodes}

Il y a d'autres méthodes fondées sur la pénétration de fluide dans les pores telles que :

- la méthode de condensation capillaire BJH (Barrett, Joyner, Halenda [15]) plutôt adaptée aux mésopores (diamètre de pore entre $2 \mathrm{~mm}$ et $50 \mathrm{~nm}$ );

- les méthodes intrusives dont la porosimétrie au mercure [16] (liquide non mouillant pour la plupart des surfaces). Elle consiste, après avoir placé l'échantillon sous vide, à faire pénétrer du mercure sous pression dans les pores (plutôt adaptée aux méso- et macropores). C'est une méthode destructive.

- la méthode à pression maximum de bulle qui n'est applicable qu'aux matériaux consolidés et utilisés sous forme de film mince (par exemple membrane filtrante). Elle consiste à saturer les pores par un liquide (généralement de l'eau) et à augmenter la pression d'air sur une des faces de la membrane jusqu'à apparition de bulles d'air sur l'autre face. Elle permet, entre autres, de localiser des inhomogénéités de taille ou de répartition des pores.

Il y aussi des méthodes calorimétriques telles que :

- la calorimétrie d'immersion fondée sur la variation d'enthalpie qui apparait quand on immerge un échantillon «sec » dans un liquide mouillant;

- la calorimétrie d'adsorption d'argon ou d'azote ou d'adsorption de liquide (par exemple, on détermine l'enthalpie de déplacement d'un alcane par un alcool); 
- la thermoporométrie [17] qui est une méthode permettant de déterminer la taille des pores en se basant sur le point de fusion ou de cristallisation d'un liquide confiné dans un pore.

On peut aussi utiliser des techniques de mesure particulières telles :

- la chromatographie liquide à haute pression (HPLC) utilisée pour évaluer la méso et macroporosité de polymères gonflants ;

- la résonnance magnétique nucléaire $(\mathrm{RMN})$ qui utilise la diffusion de molécules de ${ }^{129} \mathrm{Xe}$ dans la structure des pores. Cette méthode est bien adaptée au cas des micropores (zéolites, silices microporeuses, alumines, polymères...) ;

- une méthode ultrasonique utilisant l'atténuation et la vitesse de propagation d'ultrason dans les solides poreux. L'atténuation dépend de la taille et la distribution des pores.

\section{Normes et méthodes mises en œuvre}

En science des matériaux, on emploie le terme «éprouvette» pour désigner un échantillon de dimensions normalisées qui est soumis à un essai mécanique. C'est le terme qui est utilisé dans les deux normes dont il est question ci-après. Mais dans l'article, le terme «échantillon » est privilégié pour parler des spécimens étudiés de roche calcaire dont les dimensions ne sont pas normalisées au sens strict et ne respectent pas les préconisations des normes en question.

Il existe deux normes qui décrivent une technique gravimétrique similaire pour déterminer la porosité de roches par une méthode gravimétrique.

\subsection{Norme NF P 94-410-3}

La norme française spécifique à la détermination des propriétés physiques des roches est la norme NF P 94-410 de 2001. Elle comporte trois parties :

- NF P 94-410-1 : Détermination de la teneur en eau pondérale - Méthode par étuvage ;

- NF P 94-410-2 : Détermination de la masse volumique - Méthodes géométriques et par immersion dans l'eau;

- NF P 94-410-3 : Détermination de la porosité.

La partie NF P 94-410-3 [18] permet de déterminer, entre autres, la porosité ouverte ou connectée à partir d'une pesée hydrostatique de l'éprouvette de roche une fois saturée par de l'eau de masse volumique connue, puis par pesées à l'état saturé et ensuite après dessiccation. Elle décrit l'appareillage nécessaire, le mode opératoire et l'expression des résultats.

\subsection{Norme NF ISO 5017}

Nous nous sommes intéressés à la norme française NF ISO 5017 du 3 mai 2013 [5]. Par rapport à la norme NF P 94-410-3, elle présente l'intérêt de décrire plus précisément les conditions de préparation des éprouvettes et la mise en œuvre du mode opératoire. Elle permet, comme la norme NF P 94-410-3, de déterminer la porosité de matériaux solides à partir d'une méthode gravimétrique avec imbibition d'eau, mais elle est établie spécifiquement pour des produits réfractaires façonnés denses.

Le terme « réfractaire » implique qu'il s'agit de matériaux autres que des métaux et alliages, dont la résistance pyroscopique est supérieure ou égale à $1500{ }^{\circ} \mathrm{C}$. Ce n'est pas le cas pour les roches calcaires. Le terme « façonné » précise qu'il s'agit de produits élaborés et livrés sous une forme définitive dans laquelle ils seront mis en œuvre (briques par exemple). On peut considérer qu'une roche calcaire, utilisée sous une forme découpée ou taillée, constitue un matériau façonné. Enfin, le terme « dense » indique que les produits doivent avoir une porosité totale inférieure à $45 \%$ en fraction volumique. C'est le cas pour les échantillons de roche calcaire qui seront étudiés.

Pour les matériaux qui ne réagissent pas avec l'eau, le liquide pour immersion peut être de l'eau distillée ou de l'eau désionisée. Pour les matériaux qui sont susceptibles de réagir avec l'eau, un liquide organique adapté doit être utilisé. Pour les roches calcaires, on utilisera donc de l'eau.

La norme NF ISO 5017 qui est la traduction française de la norme internationale ISO 5017:2013 :

- définit la terminologie utilisée en porosimétrie ;

- décrit les moyens matériels nécessaires;

- donne quelques recommandations en termes de nombre et de forme des éprouvettes à analyser. Ainsi, elle préconise que le volume apparent d'une éprouvette doit être compris entre $50 \mathrm{~cm}^{3}$ et $200 \mathrm{~cm}^{3}$ et que le rapport entre la plus grande et la plus petite dimension ne doit pas dépasser 2:1;

- présente le mode opératoire et précisément la préparation et le conditionnement de l'éprouvette avant pesée ; en particulier, la norme précise qu'il faut sécher l'éprouvette jusqu'à ce que deux pesées successives, avant et après un séjour à l'étuve ne diffèrent pas de plus de $0,1 \%$; et que certains matériaux à fine porosité peuvent nécessiter de plus longues périodes d'évaporation et d'immersion;

- expose le principe de la mesure qui repose sur trois pesées comme dans la norme NF P 94-410-3 : la première pour déterminer la masse $m_{1}$ de l'éprouvette sèche, la deuxième pour mesurer la masse apparente $m_{2}$ en immersion dans le liquide choisi de l'éprouvette imbibée de ce même liquide et, enfin, la troisième pour obtenir la masse $m_{3}$ dans l'air de l'éprouvette imbibée du liquide considéré ; 
- donne les expressions des porosités à partir des masses apparentes $m_{1}, m_{2}$ et $m_{3}$, de la masse volumique de l'eau $\rho_{\mathrm{e}}$ et de la masse volumique absolue de l'éprouvette $\rho_{\mathrm{t}}$ comme suit :

- la masse volumique apparente $\rho_{\mathrm{b}}$ :

$$
\rho_{\mathrm{b}}=\frac{m_{1}}{m_{3}-m_{2}} \rho_{\mathrm{e}}
$$

- la porosité ouverte $\pi_{\mathrm{a}}$ :

$$
\pi_{\mathrm{a}}=100 \times \frac{m_{3}-m_{1}}{m_{3}-m_{2}}
$$

- la porosité totale $\pi_{\mathrm{t}}$ :

$$
\pi_{\mathrm{t}}=100 \times \frac{\rho_{\mathrm{t}}-\rho_{\mathrm{b}}}{\rho_{\mathrm{t}}}
$$

- la porosité fermée $\pi_{\mathrm{f}}$ :

$$
\pi_{\mathrm{f}}=\pi_{\mathrm{t}}-\pi_{\mathrm{a}} .
$$

L'expression (2) donne une valeur en pourcentage de la fraction volumique.

La norme NF ISO 5017 indique que les valeurs de porosité doivent être données à $0,1 \%$ près en fraction volumique. Elle souligne l'influence de la rugosité de surface et de la taille des pores et des grains du matériau sur l'exactitude des résultats obtenus par cette méthode de mesure. Elle précise que cette méthode est mal adaptée aux matériaux de texture grossière qui est elle-même relative à la taille de l'éprouvette. En d'autres termes, plus la texture du matériau est grossière, plus la taille de l'éprouvette doit être grande.

\subsection{Méthode utilisée pour l'étude}

Bien que les normes NF P 94-410-3 et NF ISO 5017 soient très proches dans la façon de déterminer la porosité ouverte de matériaux, le mode opératoire utilisé pour cette étude est plutôt inspirée de celle décrite dans la norme NF ISO 5017. Ce choix a été motivé par les constats suivants :

- les échantillons de roche calcaire, s'ils sont suffisamment homogènes, peuvent être assimilés à un matériau façonné dense. De plus, la méthode de détermination de la porosité ne dépend pas du caractère réfractaire du matériau;

- le mode opératoire décrit dans la norme NF ISO 5017 est beaucoup plus précis et détaillé sur certains aspects que celui de la norme NF P 94-410-3;

- enfin, la norme NF ISO 5017 est une norme française traduite de la norme ISO 5017 reconnue internationalement et établie récemment en 2013, qui s'appuie donc sur la longue expérience de plusieurs experts du domaine.

La procédure que nous avons choisie, inspirée de la norme NF ISO 5017, a été adaptée en fonction des moyens matériels disponibles au laboratoire.

\subsubsection{Détermination de la masse apparente $m_{1}$ de l'échantillon sec}

\subsubsection{Séchage de l'échantillon}

On place l'échantillon sur un support dans une chambre à vide dont les parois sont chauffées à $180^{\circ} \mathrm{C}$ au moyen d'un cordon chauffant. Avant fermeture de l'enceinte, on met en température l'échantillon à l'aide d'un pistolet thermique. L'échantillon est ainsi chauffé à environ $150{ }^{\circ} \mathrm{C}$. On ferme l'enceinte, puis on pompe pour atteindre un vide grossier $(<1 \mathrm{kPa})$. On maintient ces conditions pendant $1 \mathrm{~h}$ minimum (étuvage). Ensuite, on arrête le chauffage et on remet l'enceinte à l'air. Comme indiqué dans la norme, on doit adapter le temps de séchage jusqu'à ce que l'échantillon atteigne une valeur de masse constante.

\subsubsection{Pesage de l'échantillon sec}

On transfère rapidement l'échantillon chaud de l'enceinte sur un bloc en alliage d'aluminium de $2 \mathrm{~cm}$ d'épaisseur, placé sur le plateau de la balance. La balance est tarée initialement avec le bloc. On note la valeur de masse apparente $m_{1}$ indiquée par la balance.

\subsubsection{Détermination de la masse apparente $m_{2}$ de l'échantillon imbibé et immergé}

\subsubsection{Imbibition de l'échantillon}

On transfère l'échantillon encore chaud dans un dessiccateur/saturateur sur un support à trois plots (la nacelle de pesée a été préalablement installée dans le récipient). On met le dessiccateur sous vide grossier $(<1 \mathrm{kPa})$ pendant $1 \mathrm{~h}$ (phase ayant pour but de vider les pores de l'air qu'ils contiennent). On casse le vide par injection d'eau jusqu'à immersion complète de l'échantillon. La norme NF ISO 5017 indique que le liquide pour immersion peut être de l'eau distillée ou de l'eau désionisée, mais, pour cette étude, on a utilisé de l'eau de ville (voir Sect. 7.2.2 pour une justification de ce choix). Une fois l'échantillon complètement immergé, on l'agite pour éliminer les microbulles provenant du dégazage de l'eau et on le maintient en immersion pendant $1 \mathrm{~h}$ au minimum (phase de saturation). L'eau est renouvelée à chaque essai. La durée d'imbibition peut être adaptée en fonction de la porosité de l'échantillon.

\subsubsection{Pesée hydrostatique de l'échantillon imbibé}

On place le récipient contenant l'échantillon immergé sur une plateforme fixée à un dispositif de translation verticale sous la balance et on accroche la nacelle au dispositif de pesée hydrostatique, toujours en maintenant l'échantillon immergé. On tare la balance avec la nacelle immergée ne supportant pas l'échantillon. Puis on dépose l'échantillon sur la nacelle en déplaçant verticalement le récipient au moyen du translateur. On pèse l'échantillon ainsi immergé dans l'eau. On note la valeur de masse apparente $m_{2}$ de l'échantillon immergé et on mesure la température $t$ de l'eau pour calculer la masse volumique $\rho_{\mathrm{e}}$ de l'eau. 


\subsubsection{Détermination de la masse apparente $m_{3}$ de l'échantillon imbibé d'eau}

\subsubsection{Essuyage de l'échantillon imbibé d'eau}

On sort l'échantillon de l'eau et, pour éliminer l'excès d'eau en surface, on l'essuie sur toutes ses faces avec un linge en soie, initialement mouillé sous un flux continu d'eau et essoré à la main. On réalise cette opération rapidement pour éviter d'aspirer l'eau des pores.

\subsubsection{Pesage de l'échantillon imbibé d'eau}

On pèse l'échantillon imbibé et on note la valeur de masse apparente $m_{3}$ indiquée par la balance.

\subsubsection{Protocoles particuliers}

Deux variantes de ce mode opératoire ont été testées. Voici les principaux points caractéristiques de chacune de ces variantes.

\subsubsection{Protocole 1}

\section{Séchage et étuvage :}

Le temps de séchage à l'air libre (dans une salle climatisée à une température de $20^{\circ} \mathrm{C}$ et un taux d'humidité relative de $50 \%$ ) d'un échantillon entre deux séquences de mesure est de l'ordre de $12 \mathrm{~h}$.

Ensuite, pour favoriser la montée en température de l'échantillon placé sur un socle plan dans la chambre à vide, la chambre est d'abord chauffée par balayage au dessus de l'ouverture principale pendant au moins $5 \mathrm{~min}$ au moyen d'un pistolet thermique réglé à $300^{\circ} \mathrm{C}$ en température de sortie. Pendant cette opération, la tuyère du pistolet doit rester à une distance d'environ $10 \mathrm{~cm}$ de l'échantillon. Parallèlement, le cordon chauffant est alimenté pour atteindre environ $180^{\circ} \mathrm{C}$. L'enceinte, toujours chauffée par le cordon chauffant, est ensuite fermée et mise sous vide au moyen d'une pompe primaire.

\section{Pesée hydrostatique :}

Afin de maintenir l'échantillon toujours immergé, un support fixe à trois plots et la nacelle de pesée sont placés au fond du dessiccateur/saturateur, l'échantillon reposant sur les trois plots du support avant la pesée. Dans ce protocole, on utilise une nacelle qui présente une forte section à l'interface air/eau.

\subsubsection{Protocole 2 :}

\section{Séchage et étuvage :}

Le temps de séchage à l'air libre, dans la salle climatisée, d'un échantillon entre deux séquences de mesure est supérieur à $24 \mathrm{~h}$.

Puis, l'échantillon est placé sur un support à trois plots dans la chambre à vide afin d'augmenter la surface

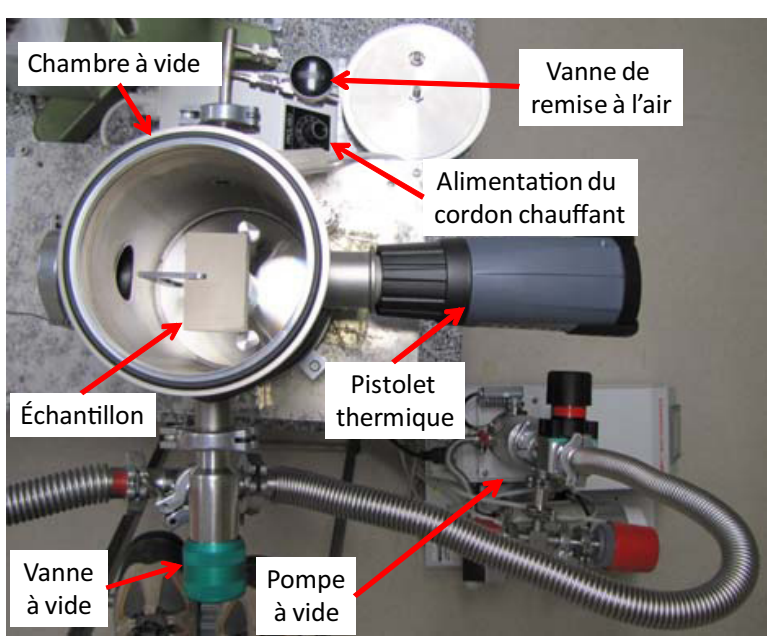

Fig. 1. - Dispositif d'étuvage ; configuration de la chambre à vide pour le chauffage de l'échantillon au moyen d'un pistolet thermique dans le cas du protocole 2 .

d'échange. Le pistolet thermique réglé à $150^{\circ} \mathrm{C}$ est inséré dans une tubulure latérale de la chambre à vide à hauteur de l'échantillon et à environ $5 \mathrm{~cm}$ de celui-ci pour améliorer l'homogénéité de chauffage de l'échantillon. De plus, le temps de chauffage au pistolet thermique est d'au moins $15 \mathrm{~min}$.

\section{Pesée hydrostatique :}

Même procédure que celle du protocole 1, mais on utilise une nacelle suspendue par un fil fin qui traverse l'interface air/eau afin de réduire les effets de capillarité et de poussée d'Archimède.

\section{Matériel}

\subsection{Dispositifs d'étuvage et d'imbibition}

Pour cette étude, un montage expérimental d'étuvage et d'imbibition a été réalisé avec les moyens disponibles au laboratoire.

Le laboratoire ne disposant pas d'étuve, le dispositif d'étuvage est constitué d'une simple enceinte à vide autour de laquelle est lové un cordon chauffant. L'enceinte est équipée d'une vanne pour l'isoler de la pompe à vide et d'une seconde vanne pour la remise à l'air. La figure 1 montre le montage lors de l'utilisation du pistolet thermique dans le cas du protocole 2 .

Le dispositif d'imbibition (fig. 2) est constitué d'un dessiccateur/saturateur en verre relié par l'intermédiaire d'une vanne trois voies à un réservoir d'eau et à une pompe à vide primaire. Un piège à gravité est placé sur le circuit de pompage pour prévenir tout risque de refoulement d'eau vers la pompe à vide. La figure 3 montre le dessiccateur/saturateur lors de la mise sous vide de l'échantillon. 


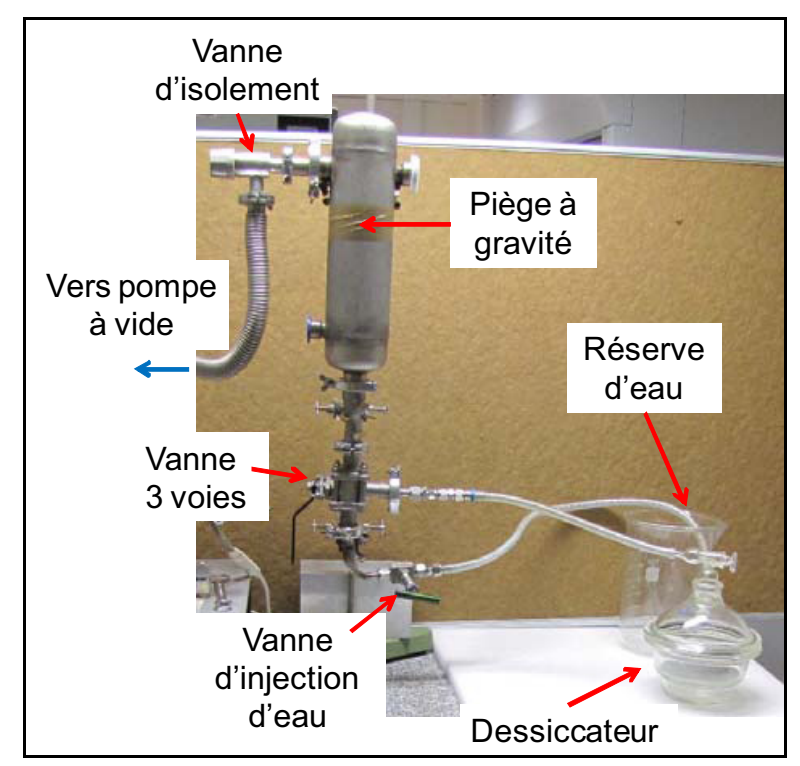

Fig. 2. - Dispositif d'imbibition.

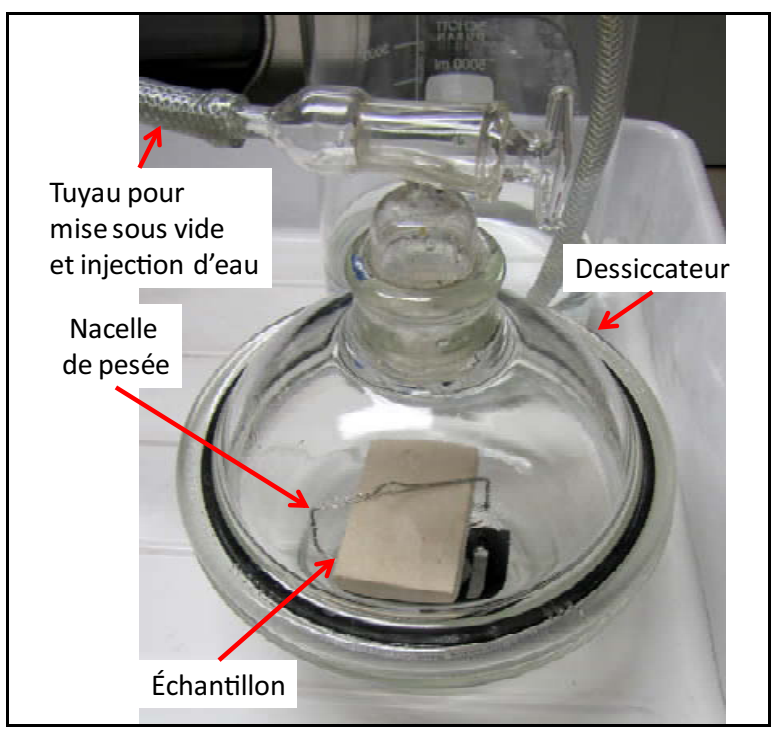

Fig. 3. - Opération de mise sous vide de l'échantillon dans le dessiccateur avec la nacelle de pesée pour le protocole 2 .

\subsection{Banc de pesée hydrostatique}

Pour les pesées dans l'air ou dans l'eau, on utilise une balance Mettler-Toledo PG4002-S Delta Range de portée $4100 \mathrm{~g}$ dont la résolution est de $10 \mathrm{mg}$ pour des masses inférieures à $800 \mathrm{~g}$. Elle dispose d'un dispositif d'accrochage sous son boîtier pour y suspendre la nacelle pour réaliser la pesée hydrostatique.

L'échantillon immergé repose sur un socle à trois plots posé au fond du bol du dessiccateur (voir fig. 4), la nacelle de pesée étant insérée avant de positionner l'échantillon. Le dépôt et le retrait de l'échantillon sur la nacelle de pesée sont réalisés par le déplacement vertical du récipient au moyen d'un dispositif manuel à crémaillère (fig. 5). La température de l'eau est mesurée à l'aide d'un thermocouple connecté à un transmetteur/convertisseur lui-même connecté à un

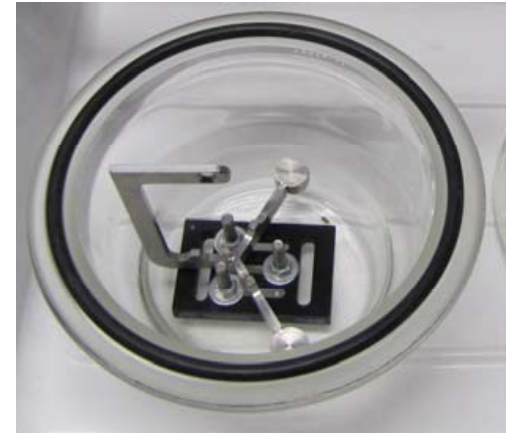

Fig. 4. - Bol du dessiccateur/saturateur avec le support à trois plots et la nacelle de pesée utilisée pour le protocole 1 .

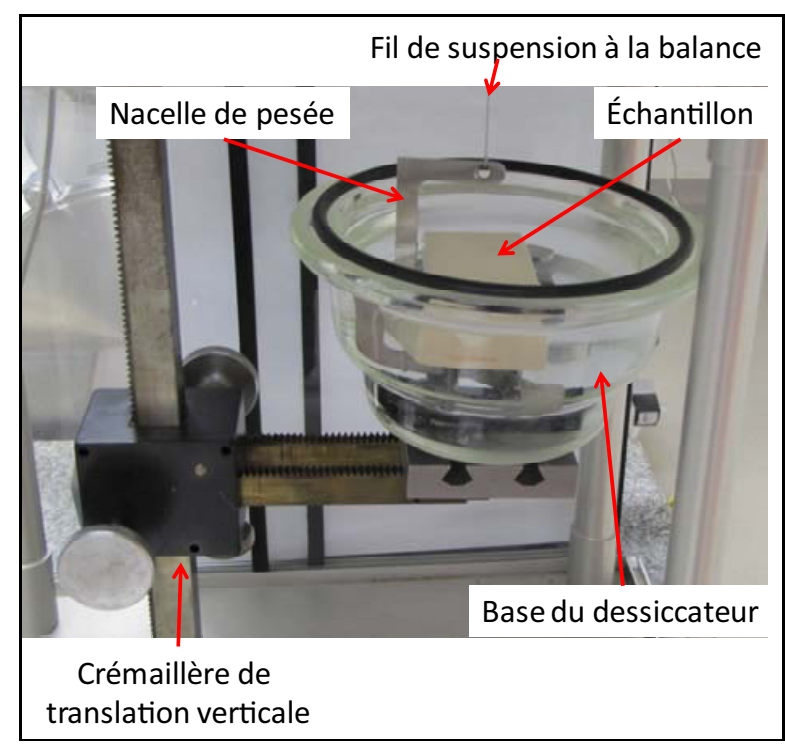

Fig. 5. - Dessiccateur/saturateur installé sous la balance pour la pesée hydrostatique dans le cas du protocole 1 .

multimètre affichant directement la température en degrés Celsius.

\section{5. Échantillons}

Tous les échantillons proviennent d'une même grande plaque de calcaire dont l'épaisseur est de $2 \mathrm{~cm}$, mais ont été prélevés à différents endroits de la plaque en fonction de l'apparence superficielle de la plaque. Les caractéristiques des échantillons sont les suivantes :

- nature : calcaire naturel ;

- apparence : quelques macrocavités, empreintes et inclusions de fossiles ;

- dimensions approximatives : $2 \mathrm{~cm} \times 5 \mathrm{~cm} \times 8 \mathrm{~cm}$;

- masse approximative : $180 \mathrm{~g}$.

Compte tenu de l'épaisseur initiale de la plaque ainsi que de la masse (proche de $200 \mathrm{~g}$ ) et du volume souhaités (proche de $100 \mathrm{~cm}^{3}$ ) pour chaque échantillon, il n'a pas été possible de respecter la recommandation de la norme qui préconise que le rapport entre la plus grande et la plus 


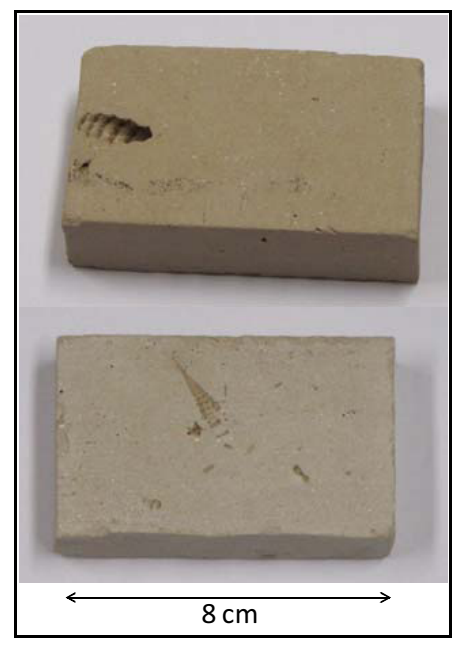

Fig. 6. - Photographie des deux faces principales de l'échantillon $\mathrm{n}^{\circ} 1$ de calcaire.

petite dimension ne doit pas dépasser 2:1. L'épaisseur des échantillons étant fixée, leurs deux autres dimensions résultent d'un compromis entre masse et volume permettant d'obtenir la meilleure incertitude relative sur la détermination de la différence de masse $m_{3}-m_{1}$.

\section{1. Échantillon $n^{\circ} 1$}

L'échantillon $\mathrm{n}^{\circ} 1$ est d'apparence peu poreuse avec une surface relativement lisse et brillante, mais des macrocavités (empreintes de fossile) sont bien visibles en surface (fig. 6). Ce n'est pas le genre d'échantillon idéal pour effectuer une mesure de porosité. En effet, il peut rester des gouttes d'eau logées dans ces macrocavités lors de la pesée de $m_{3}$ occasionnant une erreur de mesure difficilement quantifiable. La norme NF ISO 5017 préconise dans sa Section 6.4 d'éliminer tout échantillon présentant des fissures qui pourraient causer des erreurs dans la détermination du volume apparent. Dans le cas de l'échantillon $\mathrm{n}^{\circ} 1$, ces macrocavités peuvent entraîner ce type d'erreurs, cet échantillon n'a donc pas été retenu pour effectuer des mesures.

\section{2. Échantillons $n^{\circ} 2$ et $n^{\circ} 3$}

Les échantillons $\mathrm{n}^{\circ} 2$ et $\mathrm{n}^{\circ} 3$, qui ont été choisis pour déterminer leur porosité ouverte, présentent une surface assez homogène sans fissures ni macrocavités, mais leur aspect superficiel est assez différent bien que ces échantillons proviennent de la même plaque de calcaire. L'apparence visuelle et la sensation au toucher de la surface de l'échantillon $n^{\circ} 2$ révèlent le caractère rugueux de sa surface alors que celles de l'échantillon $n^{\circ} 3$ montrent un aspect brillant et lisse.

La figure 7 présente les images de leur surface prise au microscope optique avec un éclairage rasant. Sans réaliser une analyse stéréologique, on a effectué un traitement numérique simple de ces images consistant à en extraire une représentation binaire noir et blanc et un graphique du niveau moyen de gris (proportion de noir
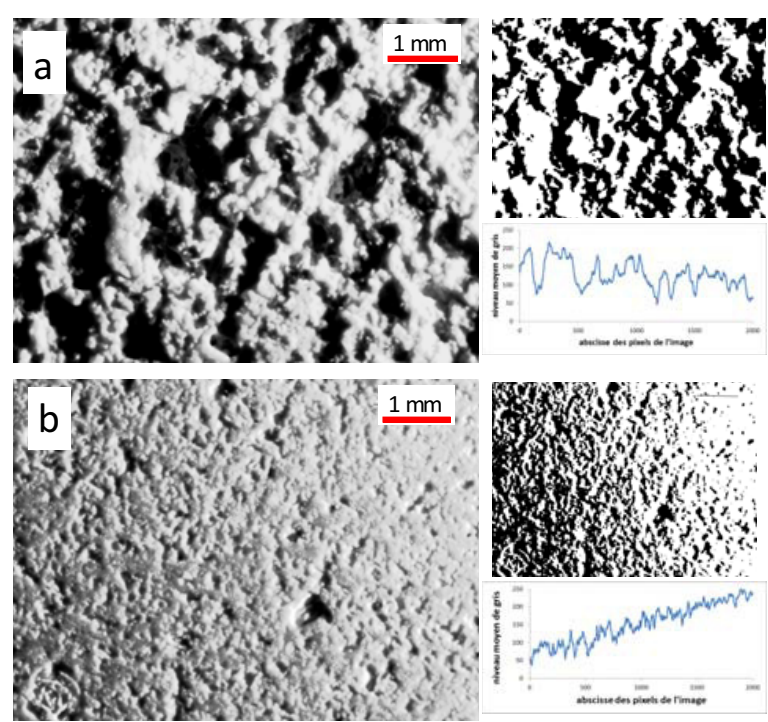

Fig. 7. - À gauche, images prises au microscope optique de la surface de l'échantillon $n^{\circ} 2$ (a) et de l'échantillon $n^{\circ} 3$ (b) ; à droite, images traitées numériquement en binaire $\mathrm{N} \& \mathrm{~B}$ inversé et représentations du niveau moyen de gris.

et de blanc) par colonne de pixels. Ces représentations donnent des informations qualitatives concernant la rugosité relative des deux surfaces ainsi que le rapport de surface entre les pores et la matrice, rapport lié à la taille relative des pores. Cependant, il est impossible à partir de ces images d'évaluer la taille relative des pores entre ces deux échantillons.

Les graphiques présentent une pente générale, exprimée en niveau de gris par abscisse de pixels, de -0,030 9 pour l'échantillon $\mathrm{n}^{\circ} 2$ et 0,0879 pour l'échantillon $\mathrm{n}^{\circ}$ 3. Ceci est dû, entre autres, à l'éclairage rasant. Ainsi, l'échantillon $\mathrm{n}^{\circ} 3$ ayant une surface relativement lisse et brillante, cela entraîne une saturation vers le blanc de l'image qui décroît depuis le côté où se trouve l'éclairage au côté opposé. Après correction de pente, on peut calculer la hauteur quadratique moyenne des graphiques qui est corrélée à la répartition des aspérités. On obtient une valeur de l'ordre de 32 pour l'échantillon $\mathrm{n}^{\circ} 2$ et de 15 pour l'échantillon $n^{\circ} 3$. Cela signifie que la répartition des aspérités est plus large pour le premier que pour le second, ce que l'on peut constater visuellement. Il existe probablement une relation de ces valeurs avec la rugosité de surface, la taille et la répartition des pores.

\section{Résultats de mesure}

\subsection{Justification des expressions de la norme NF ISO 5017}

On peut exprimer les masses apparentes sous la forme suivante :

$$
\begin{aligned}
& m_{1} \approx m-V \rho_{\mathrm{a}} \\
& m_{2} \approx m-V \rho_{\mathrm{e}} \\
& m_{3} \approx m_{\mathrm{w}}-V_{\mathrm{w}} \rho_{\mathrm{a}}
\end{aligned}
$$


avec $m$ et $V$ la masse et le volume de l'échantillon sec, $\rho_{\mathrm{a}}$ la masse volumique de l'air et $V_{\mathrm{w}}$ le volume de l'échantillon imbibé d'eau (volume apparent de l'échantillon). Le volume $V$ est la somme du volume du matériau solide $V_{\mathrm{s}}$ et du volume des pores fermés $V_{\mathrm{f}}$ :

$$
V=V_{\mathrm{s}}+V_{\mathrm{f}}
$$

La masse $m_{\mathrm{w}}$ et le volume $V_{\mathrm{w}}$ de l'échantillon imbibé d'eau ont pour expression :

$$
\begin{aligned}
& m_{\mathrm{w}}=m+V_{\mathrm{o}} \rho_{\mathrm{e}} \\
& V_{\mathrm{w}}=V_{\mathrm{s}}+V_{\mathrm{f}}+V_{\mathrm{o}}
\end{aligned}
$$

où $V_{\mathrm{o}}$ est le volume des pores ouverts.

On peut vérifier les expressions (1) à (4) données dans la norme NF ISO 5017. Pour cela, on utilise les expressions (5) à (8) en faisant l'hypothèse qu'il n'y a aucune interaction entre l'eau et la surface des pores et que l'eau pénètre complètement tous les pores ouverts de l'échantillon.

La masse volumique apparente théorique $\rho_{\mathrm{bt}}$ a pour expression :

$$
\rho_{\mathrm{bt}}=\frac{m}{V_{\mathrm{w}}} .
$$

On obtient un ordre de grandeur de l'erreur relative $e$ sur l'expression (1) tel que :

$$
\begin{aligned}
e & =\frac{\rho_{\mathrm{b}}-\rho_{\mathrm{bt}}}{\rho_{\mathrm{bt}}}=\frac{m_{1}}{m_{3}-m_{2}} \rho_{\mathrm{e}} \\
& =\left(\frac{m-V \rho_{\mathrm{a}}}{V_{\mathrm{w}}\left(\rho_{\mathrm{e}}-\rho_{\mathrm{a}}\right)} \rho_{\mathrm{e}}-\frac{m}{V_{\mathrm{w}}}\right) \frac{V_{\mathrm{w}}}{m} \\
& \approx\left(1-\frac{\rho_{\mathrm{e}}}{\rho_{\mathrm{t}}}\right)\left(1+\frac{\rho_{\mathrm{a}}}{\rho_{\mathrm{e}}}\right) \frac{\rho_{\mathrm{a}}}{\rho_{\mathrm{e}}} \approx \frac{\rho_{\mathrm{a}}}{\rho_{\mathrm{e}}} .
\end{aligned}
$$

On pourra donc apporter une correction $c_{\rho}$ telle que :

$$
c_{\rho}=-\frac{\rho_{\mathrm{a}}}{\rho_{\mathrm{e}}} \rho_{\mathrm{bt}} \approx-0,1 \% \times \rho_{\mathrm{b}} .
$$

On peut démontrer que l'expression (2) de la porosité ouverte $\pi_{\mathrm{a}}$ n'est pas biaisée :

$$
\pi_{\mathrm{a}}=100 \times \frac{m_{3}-m_{1}}{m_{3}-m_{2}}=100 \times \frac{V_{\mathrm{o}}}{V_{\mathrm{w}}} .
$$

Par contre, la porosité totale $\pi_{\mathrm{t}}$ et la porosité fermée $\pi_{\mathrm{f}}$ sont doublement biaisées, d'une part, par l'emploi de l'expression (1) donnant la masse volumique apparente et, d'autre part, par le fait que, dans le cas de cette étude, l'on ne connaisse pas précisément la masse volumique $\rho_{\mathrm{t}}$ de la partie solide du calcaire :

$$
\pi_{\mathrm{t}}=100 \times \frac{\rho_{\mathrm{t}}-\rho_{\mathrm{b}}}{\rho_{\mathrm{t}}} \approx 100 \times \frac{V_{\mathrm{f}}+V_{\mathrm{o}}}{V_{\mathrm{w}}},
$$

avec $\rho_{\mathrm{t}}$ la masse volumique de la partie solide du calcaire telle que :

$$
\rho_{\mathrm{t}} \approx \frac{m}{V_{\mathrm{s}}} \text {. }
$$

Tableau 1

Résultats des essais effectués sur l'échantillon $n^{\circ} 2$.

\begin{tabular}{|c|c|c|c|c|c|}
\hline Protocole & & $m_{1}(\mathrm{~g})$ & $m_{2}(\mathrm{~g})$ & $m_{3}(\mathrm{~g})$ & $t\left({ }^{\circ} \mathrm{C}\right)$ \\
\hline \multirow{2}{*}{1} & Moyenne & 151,72 & $94,05^{*}$ & 180,60 & 21,9 \\
\cline { 2 - 6 } & Ecart type & 0,15 & 0,11 & 0,10 & 0,7 \\
\hline \multirow{2}{*}{2} & Moyenne & 151,44 & 94,61 & 181,13 & 22,6 \\
\cline { 2 - 6 } & Ecart type & 0,01 & 0,08 & 0,09 & 0,1 \\
\hline
\end{tabular}

* Valeur corrigée pour tenir compte du niveau d'immersion de la nacelle du protocole 1 .

Tableau 2

Résultats d'essais effectués sur l'échantillon $\mathrm{n}^{\circ} 3$.

\begin{tabular}{|c|c|c|c|c|c|}
\hline Protocole & & $m_{1}(\mathrm{~g})$ & $m_{2}(\mathrm{~g})$ & $m_{3}(\mathrm{~g})$ & $t\left({ }^{\circ} \mathrm{C}\right)$ \\
\hline \multirow{2}{*}{1} & Moyenne & 176,42 & $108,43^{*}$ & 185,41 & 21,9 \\
\cline { 2 - 6 } & Ecart type & 0,03 & 0,27 & 0,25 & 1,0 \\
\hline \multirow{2}{*}{2} & Moyenne & 176,28 & 108,72 & 185,69 & 22,8 \\
\cline { 2 - 6 } & Ecart type & 0,01 & 0,13 & 0,13 & 0,2 \\
\hline
\end{tabular}

* Valeur corrigée pour tenir compte du niveau d'immersion de la nacelle du protocole 1 .

Enfin, la porosité fermée est donnée par :

$$
\pi_{\mathrm{f}}=\pi_{\mathrm{t}}-\pi_{\mathrm{a}} \approx 100 \times \frac{V_{\mathrm{f}}}{V_{\mathrm{w}}} .
$$

La masse volumique $\rho_{\mathrm{t}}$ de la partie solide du calcaire devrait être comprise entre $2,68 \mathrm{~g} \cdot \mathrm{cm}^{-3}$ et $2,76 \mathrm{~g} \cdot \mathrm{cm}^{-3} \mathrm{se}-$ lon les données bibliographiques. On peut prendre $(2,72 \pm$ $0,05) \mathrm{g} \cdot \mathrm{cm}^{-3}$. Compte-tenu de l'incertitude sur la valeur de la masse volumique de la matrice de calcaire, on ne cherche pas, dans cet article, à calculer ni à analyser les valeurs de la porosité totale $\pi_{\mathrm{t}}$ et de la porosité fermée $\pi_{\mathrm{f}}$.

\subsection{Résultats pour les échantillons $n^{\circ} 2$ et $n^{\circ} 3$}

\subsubsection{Mesures}

On a vérifié que la variation relative de masse volumique de l'air $\rho_{\mathrm{a}}$ durant chaque essai est restée dans un intervalle de $\pm 5 \%$ autour de sa valeur conventionnelle $1,2 \times 10^{-3} \mathrm{~g} \cdot \mathrm{cm}^{-3}$, et que la température de l'eau de pesée hydrostatique est restée constante à mieux que $\pm 1{ }^{\circ} \mathrm{C}$ durant chaque essai. La température étant voisine de $22{ }^{\circ} \mathrm{C}$, on pourra prendre en première approximation $0,998 \mathrm{~kg} \cdot \mathrm{m}^{-3}$ pour la valeur de la masse volumique $\rho_{\mathrm{e}}$ de l'eau.

Les tableaux 1 et 2 donnent la moyenne et l'écart type sur la moyenne (c'est-à-dire la composante d'incertitude de mesure évaluée par une méthode de type A) de cinq séries de mesures effectuées dans des conditions de répétabilité pour les échantillons $n^{\circ} 2$ et $n^{\circ} 3$ respectivement en fonction du protocole mis en œuvre.

Pour le protocole 1, on a appliqué une correction sur les mesures de la masse $m_{2}$ (valeur marquée d'un astérisque dans les tabs. 1 et 2). Cette correction a pour but de tenir compte du fait que, pour poser l'échantillon sur la nacelle ou le retirer, on doit déplacer le récipient. Le niveau d'immersion de la nacelle est donc différent 
Tableau 3

Masse volumique apparente et porosité ouverte calculées pour l'échantillon $n^{\circ} 2$.

\begin{tabular}{|c|c|c|c|}
\hline Protocole & & $\rho_{\mathrm{b}}\left(\mathrm{g} \cdot \mathrm{cm}^{-3}\right)$ & $\pi_{\mathrm{a}}(\%)$ \\
\hline \multirow{2}{*}{1} & Moyenne & 1,7500 & 33,37 \\
\cline { 2 - 4 } & Ecart type & 0,0019 & 0,15 \\
\hline \multirow{2}{*}{2} & Moyenne & 1,7472 & 34,32 \\
\cline { 2 - 4 } & Ecart type & 0,0005 & 0,09 \\
\hline
\end{tabular}

Tableau 4

Masse volumique apparente et porosité ouverte calculées pour l'échantillon $\mathrm{n}^{\circ} 3$.

\begin{tabular}{|c|c|c|c|}
\hline Protocole & & $\rho_{\mathrm{b}}\left(\mathrm{g} \cdot \mathrm{cm}^{-3}\right)$ & $\pi_{\mathrm{a}}(\%)$ \\
\hline \multirow{2}{*}{1} & Moyenne & 2,2879 & 11,68 \\
\cline { 2 - 4 } & Ecart type & 0,0002 & 0,34 \\
\hline \multirow{2}{*}{2} & Moyenne & 2,2859 & 12,23 \\
\cline { 2 - 4 } & Ecart type & 0,0005 & 0,18 \\
\hline
\end{tabular}

lorsque l'on fait le zéro de la balance sans échantillon et lorsque l'on fait la pesée avec l'échantillon sur la nacelle. La base du dessiccateur n'étant pas assez profonde pour avoir cette interface au niveau du fil de suspension entre la balance et la nacelle, cette différence d'immersion joue sur la tige verticale de section rectangulaire de la nacelle (section $4 \mathrm{~mm} \times 10 \mathrm{~mm}$ ) à l'interface air/eau (fig. 5). La différence de poussée de l'eau par rapport à la hauteur d'immersion vaut $0,04 \mathrm{~g} \cdot \mathrm{mm}^{-1}$. La variation de hauteur a été estimée de l'ordre de $5 \mathrm{~mm}$ pour le protocole 1 , soit une correction sur la valeur de $m_{2}$ de $-0,2 \mathrm{~g}$.

On peut remarquer que la masse d'eau contenue dans les pores ouverts, correspondant à la différence $m_{3}-m_{1}$, est de l'ordre de $30 \mathrm{~g}$ pour l'échantillon $\mathrm{n}^{\circ} 2$ à large porosité, mais seulement de l'ordre de 10 g pour l'échantillon n 3 à fine porosité.

\subsubsection{Calcul de la masse volumique apparente et de la porosité ouverte}

À partir des résultats de pesée, les valeurs de masse volumique apparente et de porosité ouverte sont directement calculées selon les expressions (1) et (2). Les tableaux 3 et 4 donnent les valeurs moyennes et écarts types sur la moyenne des cinq valeurs calculées à partir de chaque série de mesures de masse pour les échantillons $n^{\circ} 2$ et $n^{\circ} 3$ respectivement. Les valeurs moyennes de masse volumique apparente des échantillons ont été corrigées en appliquant la correction $c_{\rho}$ donnée par l'expression (11)

À titre indicatif, on obtiendrait une porosité totale $\pi_{\mathrm{t}}$ de l'ordre de $36 \%$ et de $16 \%$, correspondant à une porosité fermée $\pi_{\mathrm{f}}$ de l'ordre de $2 \%$ et $4 \%$, pour les échantillons $n^{\circ} 2$ et $n^{\circ} 3$ respectivement. Ces valeurs sont à considérer avec circonspection. La principale composante d'incertitude provient de la connaissance de la masse volumique $\rho_{\mathrm{t}}$ de la matrice de calcaire.

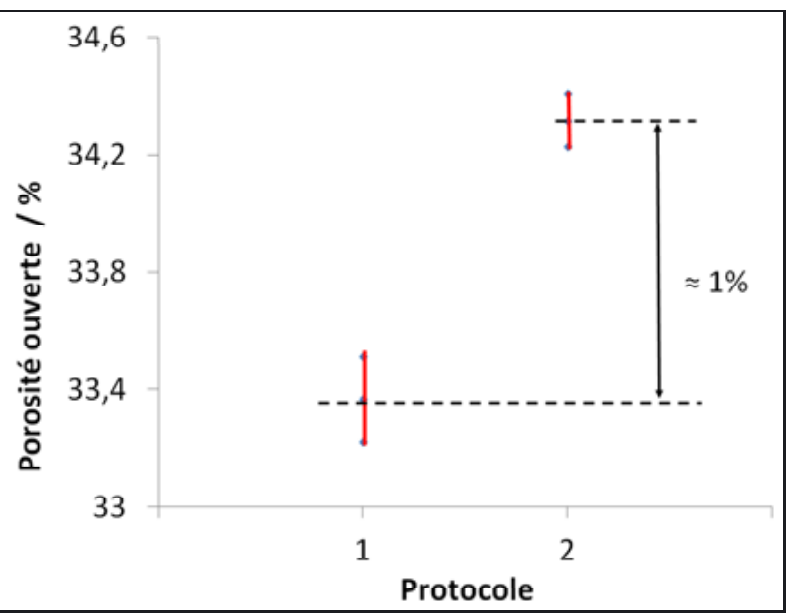

Fig. 8. - Valeur moyenne et écart type sur la moyenne de la porosité ouverte pour les séries d'essais sur l'échantillon $n^{\circ} 2$ ( 5 mesures pour chaque protocole).

Le coefficient de sensibilité $a$ de $\rho_{\mathrm{t}}$ pour $\pi_{\mathrm{t}}$ a pour expression :

$$
a=100 \times \frac{\rho_{\mathrm{b}}}{\rho_{\mathrm{t}}^{2}} .
$$

L'incertitude type sur $\rho_{\mathrm{t}}$ étant de $0,025 \mathrm{~g} \cdot \mathrm{cm}^{-3}$, on obtient une composante d'incertitude élargie $(k=2)$ sur la détermination de $\pi_{\mathrm{t}}$ de l'ordre de $1,6 \%$ et $1,2 \%$, soit une incertitude relative de $80 \%$ et $30 \%$ pour les échantillons $\mathrm{n}^{\circ} 2$ et $\mathrm{n}^{\circ} 3$ respectivement. À cela s'ajoutent les erreurs systématiques qui peuvent affecter la détermination de $\rho_{\mathrm{b}}$.

\section{7. Évaluation de l'incertitude de détermination de la porosité ouverte et discussions sur la méthode}

\subsection{Composantes d'incertitude évaluées par une méthode de type $A$}

Comme pour tout mesurage, la variabilité des résultats de mesure provient à la fois d'erreurs aléatoires et d'erreurs systématiques. Les écarts types donnés dans les tableaux 1 et 2 constituent les composantes d'incertitude évaluées par une méthode de type A, c'est-à-dire provenant d'une analyse statistique de séries de mesures effectuées dans des conditions de répétabilité.

La figure 8 présente la valeur moyenne et l'écart type sur la moyenne de la porosité ouverte déterminés à partir de séries d'essais sur l'échantillon $n^{\circ} 2$ en appliquant les protocoles 1 et 2 (valeurs du tab. 3). Comparativement au protocole 1 , le protocole 2 conduit à une réduction non seulement des écarts types, c'est-à-dire de la contribution des erreurs aléatoires, mais aussi, de manière plus significative, des erreurs systématiques, ce qui engendre une augmentation de l'ordre de $1 \%$ de la valeur moyenne de la porosité ouverte.

La figure 9 présente les résultats de la porosité ouverte obtenus sur l'échantillon $n^{\circ} 3$ (valeurs du tab. 4). 


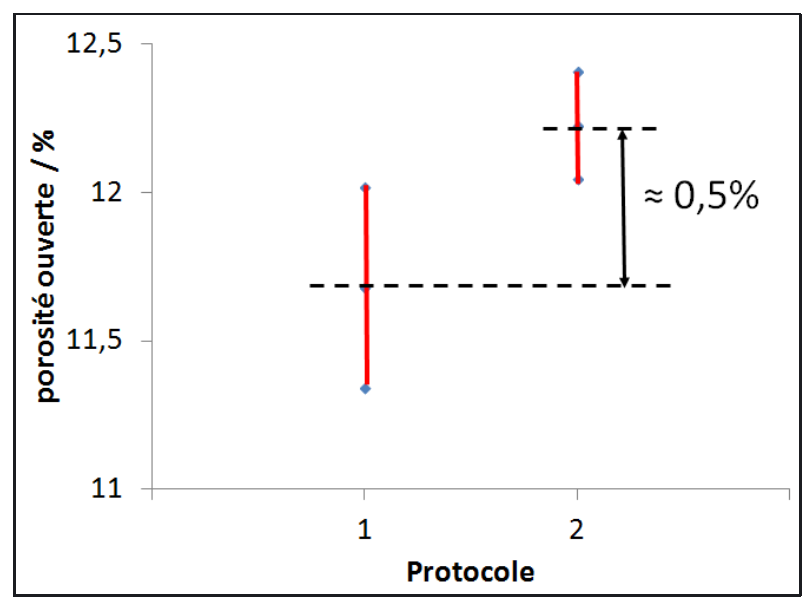

Fig. 9. - Valeur moyenne et écart type sur la moyenne de la porosité ouverte pour les séries de mesure de l'échantillon $\mathrm{n}^{\circ} 3$ ( 5 mesures pour chaque protocole).

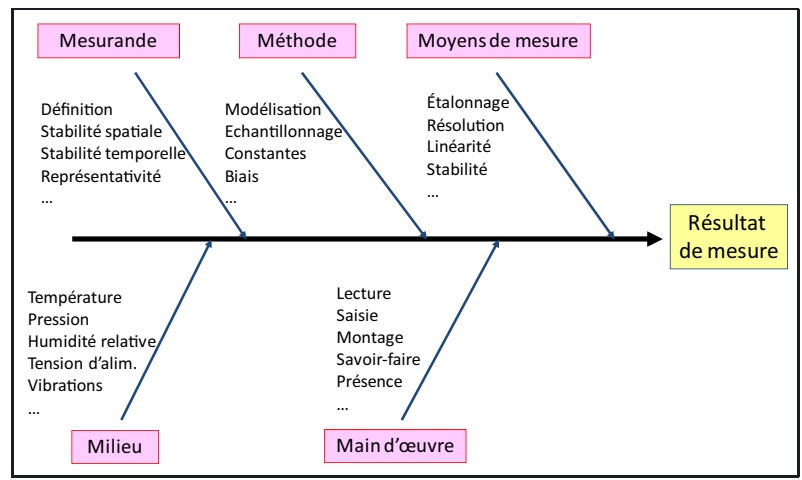

Fig. 10. - Exemple de diagramme des causes d'erreur utilisé dans la méthode des $5 \mathrm{M}$ pour l'analyse d'un processus de mesure.

On peut faire les mêmes observations que dans le cas de la figure 8 en notant cependant que l'augmentation de $0,5 \%$ de la valeur moyenne de la porosité ouverte est beaucoup moins significative devant la valeur des écarts types sur la moyenne.

Ces résultats mettent en évidence la nécessité de mener une analyse approfondie des causes d'erreur afin d'évaluer les éventuelles corrections et les composantes d'incertitude associées.

\subsection{Composantes d'incertitude évaluées par une méthode de type $B$}

Pour déterminer les sources d'erreur et estimer les composantes d'incertitude évaluées par une méthode de type $\mathrm{B}$, on utilise la méthode des 5M, couramment utilisée en métrologie, pour analyser le processus de mesure que l'on peut schématiser par le diagramme des causes d'erreur (diagramme d'Ishikawa) tel que l'exemple donné en figure 10. C'est une approche méthodique qui permet de donner un canevas d'analyse systématique permettant de définir les principales sources d'erreur de mesure, d'évaluer les éventuelles corrections à appliquer et d'estimer les composantes d'incertitude associées.

Les cinq entrées du diagramme sont analysées comme il suit.

\subsubsection{Mesurande}

Les trois échantillons découpés dans une plaque de grande dimension ne sont évidemment pas représentatifs pour déterminer la porosité moyenne de la plaque considérée. Ils sont pris, dans cette étude, comme des objets d'essai indépendants. Leur représentativité n'a donc pas à être prise en compte dans cette analyse des causes d'erreur.

L'inhomogénéité de l'échantillon (macrocavités ouvertes, fissures...) peut fausser la mesure de porosité ouverte (voir partie 3.1). On a donc écarté les échantillons présentant des macrodéfauts visibles en surface comme l'échantillon $n^{\circ} 1$. Cependant aucun des échantillons disponibles n'ayant une surface parfaitement homogène, seuls les deux échantillons offrant la meilleure homogénéité de surface ont été retenus. On admet que l'effet d'inhomogénéité de surface n'entraîne que des erreurs aléatoires.

La forme parallélépipédique aplatie de l'échantillon peut entraîner un biais. En particulier pour l'immersion, l'échantillon est placé de telle façon que ses deux plus grandes faces soient horizontales. Cela doit être pris en compte lorsqu'il y a apparition de bulles dans l'eau qui peuvent rester prisonnières sous la face inférieure $\left(\approx 40 \mathrm{~cm}^{2}\right)$ de l'échantillon.

Pour l'échantillon à large porosité et par conséquent à surface rugueuse, des particules solides pourraient être arrachées de la surface au cours des manipulations. On admet que cet échantillon a été manipulé avec suffisamment de précaution pour ne pas entraîner de perte de matière solide appréciable.

\subsection{2. $\quad$ Méthode}

\subsubsection{Préparation de l'échantillon}

\section{Le séchage :}

La condition pour obtenir un échantillon sec constitué d'un matériau à fine porosité après une séquence de détermination de sa porosité, c'est-à-dire pour que sa masse reste constante à $0,1 \%$ après plusieurs étuvages successifs comme il est spécifié dans la partie 7.1 de la norme NF ISO 5017, est évidemment plus longue que pour un matériau à large porosité.

La figure 11 présente le pourcentage d'eau restant dans les pores à différentes étapes de la phase de séchage/étuvage correspondant au protocole 2 à l'exception de l'étape 4 qui a été effectuée ici pour vérifier que l'étape 3 était suffisante pour obtenir un séchage complet. On voit qu'après un séchage à l'air de $24 \mathrm{~h}$, il reste plus de $8 \%$ des $10 \mathrm{~g}$ d'eau dans les pores de l'échantillon 


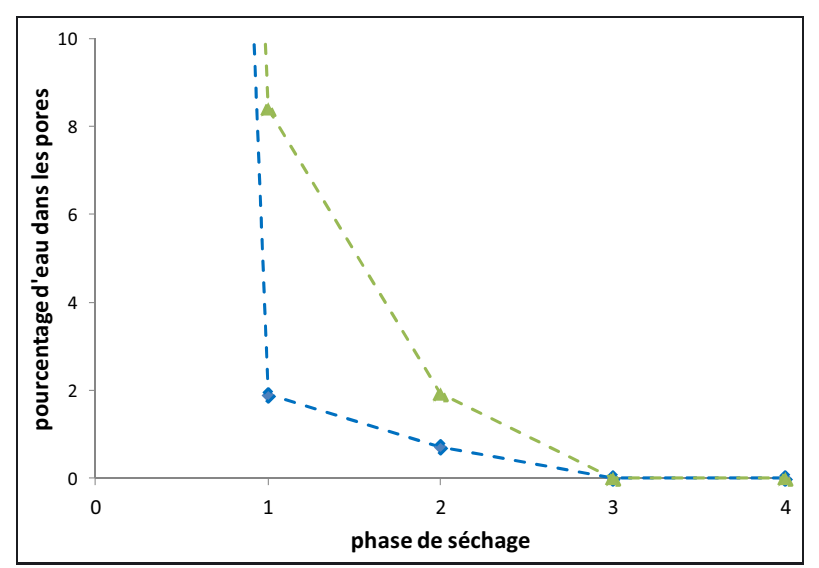

Fig. 11. - Pourcentage d'eau restant dans les pores ouverts pour l'échantillon $n^{\circ} 2$ (point losange) et pour l'échantillon $n^{\circ} 3$ (point triangulaire) à chaque étape de séchage/étuvage du protocole 2 : étape 0 : échantillon imbibé d'eau (correspondant à $100 \%$ d'eau ) ; étape 1 : séchage à l'air $\left(20^{\circ} \mathrm{C}\right.$ et $\left.50 \% \mathrm{HR}\right)$ pendant $24 \mathrm{~h}$; étape 2 : séchage au pistolet thermique à $150{ }^{\circ} \mathrm{C}$ pendant $15 \mathrm{~min}$; étape 3 : étuvage $\left(180^{\circ} \mathrm{C}\right.$ et $\left.5 \mathrm{~Pa}\right)$ pendant $1 \mathrm{~h}$; étape 4 : étuvage $\left(180^{\circ} \mathrm{C}\right.$ et $\left.5 \mathrm{~Pa}\right)$ pendant $1 \mathrm{~h}$ de plus.

$\mathrm{n}^{\circ} 3$, soit de l'ordre de $0,8 \mathrm{~g}$ alors qu'il n'en reste que $2 \%$ des $30 \mathrm{~g}$ d'eau pour l'échantillon $\mathrm{n}^{\circ} 2$, soit $0,6 \mathrm{~g}$. De plus, on constate qu'après le séchage au pistolet thermique selon le protocole 2, il reste approximativement $0,7 \%$ d'eau pour l'échantillon $\mathrm{n}^{\circ} 2$, et $2 \%$ d'eau pour l'échantillon $\mathrm{n}^{\circ} 3$, ce qui correspond à environ $0,2 \mathrm{~g}$ d'eau pour chaque échantillon. Enfin, il semble qu'un étuvage appliqué pendant une heure soit suffisant pour éliminer l'eau restante dans les pores de ces deux échantillons.

Il est évident que le protocole 2 est mieux adapté à un matériau à fine porosité que le protocole 1 . Cela est confirmé par l'examen des valeurs reportées dans les tableaux 1 et 2 . En effet, on note que le protocole 2 permet de réduire significativement les valeurs moyennes de $m_{1}$ et les écarts types associés grâce à l'amélioration du séchage de l'échantillon.

\section{L'imbibition :}

\section{- Bulles de gaz}

Un point important de la phase d'imbibition est la mise en immersion de l'échantillon qui est effectuée par l'injection d'eau dans le dessiccateur qui a été préalablement mis sous vide primaire. Quelle que soit la nature de l'eau utilisée, de nombreuses microbulles apparaissent dans l'eau et certaines d'entre elles viennent se coller sous la face inférieure de l'échantillon. Il faut donc bien agiter l'échantillon pour en éliminer une grande partie.

Des essais préliminaires de dégazage ont été réalisés avec de l'eau désionisée par osmose inverse, de l'eau distillée et de l'eau de ville. L'eau est stockée à la température de la salle climatisée à $20^{\circ} \mathrm{C} \pm 0,5^{\circ} \mathrm{C}$ dans un grand bécher. Ces essais ont consisté à injecter l'eau considérée dans le dessiccateur/saturateur préalablement mis sous vide. Le passage du circuit de pompage à celui d'injection

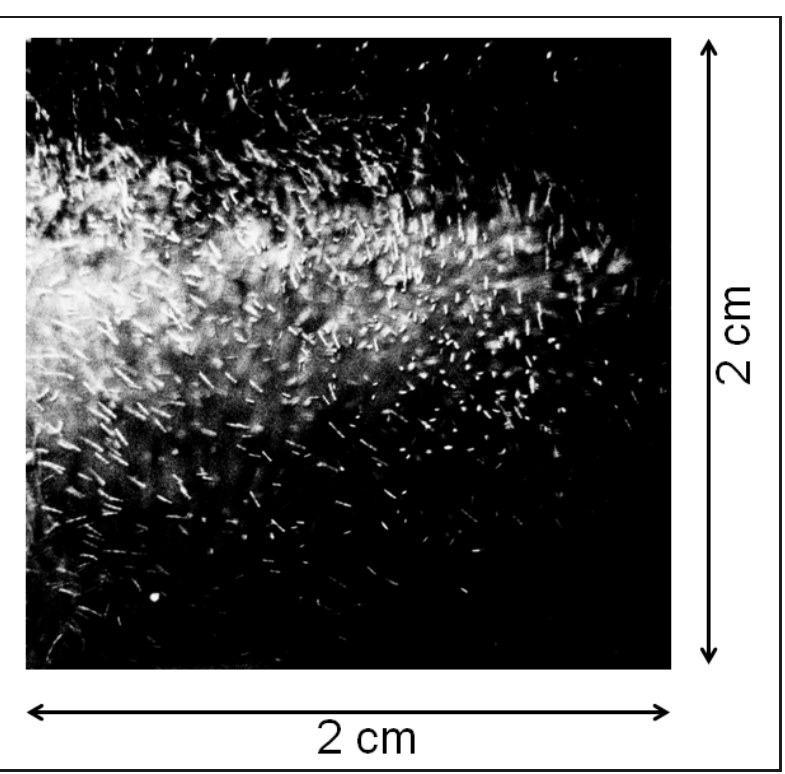

Fig. 12. - Macrophotographie couvrant un champ vertical sous la surface de l'eau (l'eau apparait en noir) au cours de l'injection d'eau dans le dessiccateur initialement sous vide. Apparition de microbulles en mouvement (points blancs).

d'eau est assuré au moyen d'une vanne trois voies. L'injection d'eau est provoquée par l'ouverture d'une vanne d'isolement, placée sur le tuyau reliant le bécher au dessiccateur, grâce à la différence entre la pression atmosphérique au dessus de l'eau du bécher et le vide grossier (environ $800 \mathrm{~Pa}$ ) qui règne dans le dessiccateur. Tous les essais ont mis en évidence l'apparition des nombreuses microbulles de l'ordre de $0,1 \mathrm{~mm}$ de diamètre dans l'eau du dessiccateur quel que soit la nature de l'eau.

Il faut préciser deux choses. Premièrement, la norme préconise d'introduire progressivement le liquide pour immersion de façon qu'après 3 min, l'éprouvette soit recouverte d'environ $20 \mathrm{~mm}$ de liquide. Cela n'est pas réalisable dans notre cas, compte tenu de la vanne utilisée, l'injection de l'eau (environ $800 \mathrm{~cm}^{3}$ ) est effectuée en une trentaine de secondes seulement. Deuxièmement, l'air contenu dans la portion de tuyau (quelques centimètres cubes) entre la vanne d'injection d'eau et la vanne trois voies ne peut pas être pompé. Lorsque l'on bascule la vanne trois voies, les quelques centimètres cubes d'air à pression atmosphérique vont se détendre dans les $1000 \mathrm{~cm}^{3} \mathrm{du}$ dessiccateur dont la pression se stabilisera à environ $2,2 \mathrm{kPa}$. Cela reste compatible avec la recommandation de la norme qui stipule que la pression doit être égale ou inférieure à $2,5 \mathrm{kPa}$.

La figure 12 présente une macrophotographie prise sous la surface de l'eau dans le dessiccateur sous basse pression au cours de l'injection d'eau. Elle montre des microbulles en mouvement dans l'eau. Ce même type d'observation a été constaté aussi bien avec de l'eau bidistillée, de l'eau désionisée et de l'eau de ville. C'est la raison pour laquelle, compte tenu de ces observations, il a été décidé de n'utiliser que de l'eau de ville. Malgré le fait d'agiter l'échantillon pour éliminer les bulles collées à la 


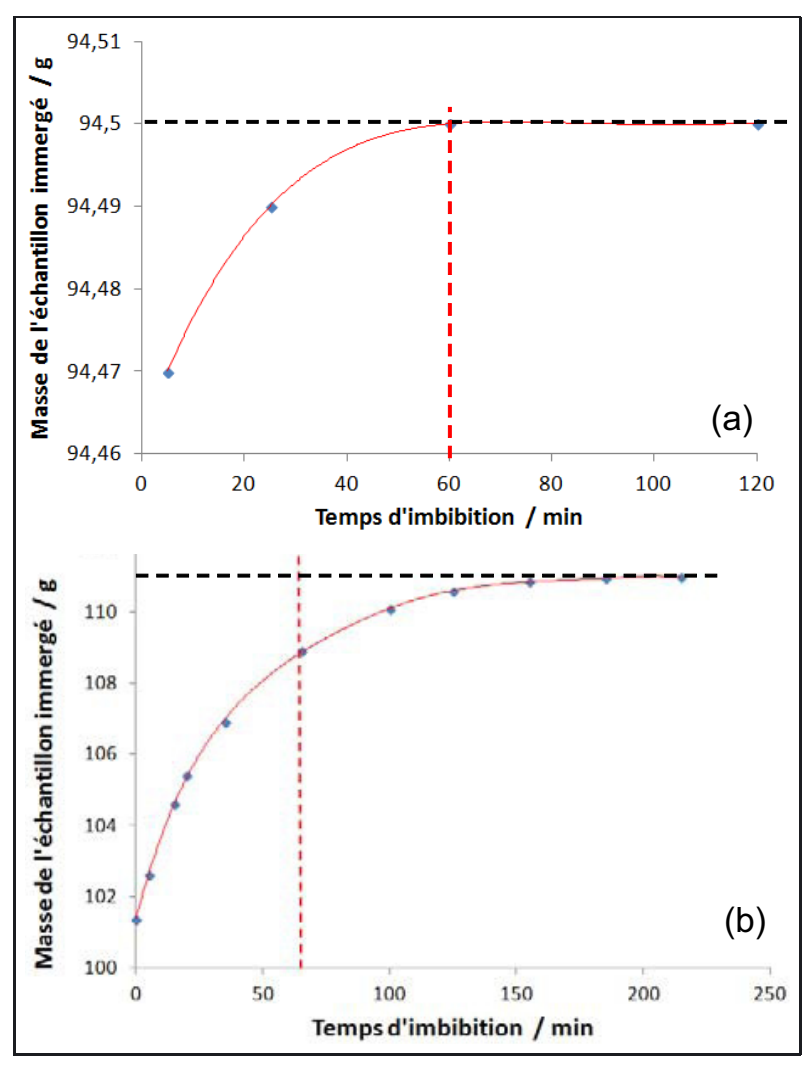

Fig. 13. - Résultats de pesée hydrostatique obtenus au cours de la phase d'imbibition de l'échantillon $\mathrm{n}^{\circ} 2$ (a) et de l'échantillon $\mathrm{n}^{\circ} 3$ (b).

face inférieure de l'échantillon, il est inévitable qu'il y ait des bulles qui restent prisonnières des aspérités et qui pénètrent dans les pores entraînant une erreur systématique sur la détermination de la porosité ouverte. Plus la surface de l'échantillon sera rugueuse et plus ce phénomène sera important et sera la source d'une erreur systématique due à l'occupation par du gaz d'un petit volume des pores ouverts empêchant l'eau de pénétrer complètement dans les pores. En considérant qu'il peut y avoir par exemple un volume de $10 \mathrm{~mm}^{3}$ d'air qui pénètre dans les pores, cela correspondrait à une masse manquante de $10 \mathrm{mg}$ d'eau sur $m_{2}$ et $m_{3}$. Pour tenir compte de cette source d'erreur non corrigée et pour les cas extrêmes, on prendra une incertitude type due à la pénétration d'air de $10 \mathrm{mg}$ sur la mesure des masses $m_{2}$ et $m_{3}$.

\section{- Le temps d'imbibition}

Un second point important de la phase d'imbibition concerne la saturation par l'eau des pores ouverts. Pour mettre en évidence le temps nécessaire à la saturation, des pesées hydrostatiques ont été effectuées durant la phase d'imbibition des échantillons jusqu'à stabilisation de leur masse.

La figure 13 représente les valeurs mesurées pour l'échantillon $n^{\circ} 2$ (fig. 13a) et pour l'échantillon $n^{\circ} 3$ (fig. 13b). On voit dans le cas de l'échantillon $n^{\circ} 3$ à fine porosité que la saturation complète des pores ouverts nécessite presque $3 \mathrm{~h}$ alors que l'échantillon $\mathrm{n}^{\circ} 2$ à large
Tableau 5

Résultats d'essais effectués sur l'échantillon $\mathrm{n}^{\circ} 1$ en utilisant le protocole 1 .

\begin{tabular}{|c|c|c|c|c|}
\hline & $m_{1}(\mathrm{~g})$ & $m_{2}(\mathrm{~g})$ & $m_{3}(\mathrm{~g})$ & $t\left({ }^{\circ} \mathrm{C}\right)$ \\
\hline Moyenne & 179,42 & $107,76^{*}$ & 186,40 & 22,7 \\
\hline Ecart type & 0,20 & 0,30 & 0,72 & 0,1 \\
\hline
\end{tabular}

* Valeur corrigée pour tenir compte du niveau d'immersion de la nacelle.

porosité est saturé d'eau au bout d'une heure d'immersion. Cette étude a été réalisée deux fois et a donné les mêmes résultats à chaque fois. Là encore, on peut s'attendre à une erreur systématique du fait de la saturation incomplète des pores ouverts pour l'échantillon $n^{\circ} 3$. Cela montre qu'aucun des deux protocoles n'est parfaitement adapté à un échantillon à fine porosité. Dans les protocoles 1 et 2, on ne laisse qu'une heure d'imbibition. Si l'on considère l'échantillon $\mathrm{n}^{\circ} 3$, la figure $13 \mathrm{~b}$ montre qu'il pourrait encore absorber environ $2 \mathrm{~g}$ d'eau. Cela conduirait à une erreur systématique de l'ordre de $20 \%$ en valeur relative sur la détermination de $m_{2}$ et $m_{3}$. On admet que cette correction est déterminée avec une erreur maximale de $\pm 0,1 \mathrm{~g}$, d'où, une incertitude type de $60 \mathrm{mg}$ en prenant une loi de distribution de probabilité uniforme.

\section{L'essuyage :}

Pour essuyer l'échantillon imbibé d'eau avant sa pesée dans l'air, la norme NF ISO 5017 préconise d'utiliser un linge en lin qu'on laisse complètement imbibé dans le liquide pour immersion et qu'on essore légèrement à la main avant chaque utilisation. Pour l'essuyage proprement dit, elle stipule d'extraire l'éprouvette du liquide et, sans attendre, d'éliminer l'excès de liquide en le roulant rapidement sur le linge humide, sur toutes les faces, puis de sécher les deux extrémités. Il faut effectuer cette action rapidement et peser immédiatement l'échantillon afin d'éviter que le liquide ne ressorte par les pores pendant l'opération d'essuyage et ne s'évapore pendant l'opération de pesée. L'essuyage de l'échantillon est donc une opération qui dépend non seulement du mode opératoire, mais aussi de l'opérateur. Bien que le mode opératoire soit un peu différent de celui préconisé dans la norme (linge de soie et essuyage), la dispersion assez faible des valeurs de pesée $m_{3}$ indique que les opérations d'essuyage et de pesée ont été réalisées de manière relativement répétable. Mais ce constat ne permet pas de garantir l'absence d'erreur systématique due à cette phase du protocole. C'est pourquoi on attribuera une incertitude type de $10 \mathrm{mg}$ sur la mesure de $m_{3}$.

\section{Exemple mettant en évidence l'influence des cavités :}

Pour illustrer ces différents points, on a effectué des mesures sur l'échantillon $\mathrm{n}^{\circ} 1$ malgré la présence de macrocavités (voir fig. 6). Les résultats sont reportés dans le tableau 5.

Ces résultats montrent que, sur un échantillon à porosité fine, présentant une porosité ouverte autour de $10 \%$ 
et ayant des macrocavités superficielles, la dispersion des mesures est :

- importante sur $m_{1}$ en raison de la difficulté de sécher correctement les cavités

- un peu plus importante sur $m_{2}$ en raison des bulles qui viennent se loger au fond des cavités et qu'on ne peut pas éliminer par agitation de l'échantillon dans l'eau ;

- encore plus importante sur $m_{3}$ car il n'est pas possible d'éliminer correctement l'excès d'eau contenu au fond des cavités lors de l'essuyage.

Ces mesures conduisent à des valeurs de porosité ouverte de l'ordre de $10 \%$ probablement sous-évaluée pour les différentes raisons évoquées précédemment. Pour un tel échantillon, le protocole 2 permettrait de réduire un peu la dispersion des valeurs de pesée $m_{1}$ et $m_{2}$, mais pas celle de $m_{3}$.

\subsubsection{Pesées}

Pour la pesée dans l'air de l'échantillon sec, on admettra que l'humidité relative de l'air a une influence négligeable et que tous les pores ouverts sont totalement accessibles à l'air. De même, pour les pesées de $m_{2}$ et $m_{3}$, on admettra que tous les pores ouverts sont totalement accessibles à l'eau.

Pour la pesée hydrostatique dans le cas du protocole 1 , on a appliqué une correction sur la valeur de $m_{2}$ de $-0,2 \mathrm{~g}$ avec une incertitude type de l'ordre de $30 \mathrm{mg}$. Une autre source d'erreur est l'effet de capillarité à l'interface air/eau sur la tige de la nacelle qui n'est pas négligeable par rapport à l'effet qu'il y aurait sur le fil de suspension comme dans le cas du protocole 2 . Il est difficile de déterminer une correction, mais on prendra une incertitude type de $10 \mathrm{mg}$.

Pour la pesée dans l'air de l'échantillon imbibé d'eau, l'eau en excès peut rester ou non dans les aspérités de surface. Plus les pores du matériau sont larges, plus sa surface est rugueuse et plus l'essuyage est délicat à réaliser. En effet, il y a non seulement le risque d'éliminer trop d'eau à l'entrée des pores mais aussi celui d'arrachement de particules solides. Inversement, plus les pores du matériau sont fins, plus sa surface est lisse et l'essuyage avec un chiffon mouillé laisse une fine pellicule d'eau sur chaque plan de sa surface géométrique. Cela peut représenter un volume de quelques millimètres cubes. En prenant par exemple $10 \mathrm{~mm}^{3}$, on obtient une erreur sur la pesée de $10 \mathrm{mg}$ et, pour inclure les cas extrêmes, on prend cette même valeur comme incertitude type due à l'excès d'eau pour cette pesée.

\subsubsection{Moyens de mesure (instruments)}

La balance électronique a été initialement vérifiée. Aucune erreur de linéarité, ni erreur de justesse n'ont été observées. Sa résolution est de $10 \mathrm{mg}$. En lui attribuant une loi de distribution de probabilité uniforme, l'incertitude type due à la quantification est de $3 \mathrm{mg}$. Elle sera prise en compte deux fois : une fois pour la mise à zéro ou le tarage de la balance et une fois pour la pesée. Son plateau est large (carré de $20 \mathrm{~cm}$ de côté) et encastré dans le corps de la balance ce qui permet de limiter les effets de convection quand on détermine $m_{1}$ alors que l'échantillon est encore chaud $\left(\approx 150{ }^{\circ} \mathrm{C}\right)$. Pour réduire la transmission de chaleur à la balance électronique lors de la pesée de $m_{1}$, un bloc séparateur en alliage d'aluminium est placé sur le plateau pour recevoir l'échantillon chaud. La balance est initialement tarée avec ce bloc.

Pour les pesées hydrostatiques, la mesure de température de l'eau est déterminée au moyen d'un thermocouple avec une incertitude type de $0,1{ }^{\circ} \mathrm{C}$.

\subsubsection{Moyens humains (opérateurs)}

L'influence de l'opérateur est primordiale dans ces deux protocoles et tout particulièrement pour l'essuyage de l'excès d'eau de l'échantillon imbibé. Cet essuyage est une opération qui dépend non seulement de l'opérateur, mais aussi de l'état de surface de l'échantillon, de la nature du chiffon et de l'état de saturation en eau du chiffon.

On peut admettre que l'écart type obtenu à partir de mesures effectuées dans des conditions de répétabilité des résultats prend en compte cette source de variabilité et qu'il n'y a pas d'erreur systématique appréciable.

\subsubsection{Milieu (grandeurs d'influence)}

Durant les $2 \mathrm{~h}$ qui séparent la première pesée des deux autres, la variation relative de masse volumique de l'air $\rho_{\mathrm{a}}$ dans une salle climatisée à $\pm 0,5{ }^{\circ} \mathrm{C}$ reste dans l'intervalle $\pm 5 \%$ autour de sa valeur conventionnelle $1,2 \times 10^{-3} \mathrm{~g} \cdot \mathrm{cm}^{-3}$, soit une variation maximale de $\pm 6 \times 10^{-5} \mathrm{~g} \cdot \mathrm{cm}^{-3}$. On néglige donc l'influence de cette variation pour chaque essai.

La température de l'eau de pesée hydrostatique reste constante à mieux que $\pm 0,2{ }^{\circ} \mathrm{C}$ durant chaque essai. La température étant comprise entre $19{ }^{\circ} \mathrm{C}$ et $23{ }^{\circ} \mathrm{C}$ pour $l^{\prime}$ ensemble des essais correspondant à une étendue de $7 \times 10^{-4} \mathrm{~g} \cdot \mathrm{cm}^{-3}$, la masse volumique $\rho_{\mathrm{e}}$ de l'eau reste donc en première approximation autour $0,9980 \mathrm{~g} \cdot \mathrm{cm}^{-3}$ avec une incertitude type de $2 \times 10^{-4} \mathrm{~g} \cdot \mathrm{cm}^{-3}$, cette incertitude englobe celle de la mesure de température. On peut donc admettre que la masse volumique de l'eau reste constante pendant chaque essai.

\subsection{Résultats et budget d'incertitude}

Compte-tenu de l'incertitude sur la détermination de la masse volumique de la matrice des échantillons, on se limite à exprimer le résultat de mesure de la porosité ouverte pour les deux échantillons et on ne considère que le protocole 2 qui est le plus proche du mode opératoire présentée dans la norme NF ISO 5017. 
Tableau 6

Composantes d'incertitude sur les pesées pour les échantillons n ${ }^{\circ} 2$ et $n^{\circ} 3$ dans le cas du protocole 2 .

\begin{tabular}{|c|c|c|c|c|c|}
\hline échantillon & \multicolumn{2}{|c|}{ composante } & $m_{1}(\mathrm{mg})$ & $m_{2}(\mathrm{mg})$ & $m_{3}(\mathrm{mg})$ \\
\hline \multirow{8}{*}{$\mathrm{n}^{\circ} 2$} & \multicolumn{2}{|c|}{$\mathrm{A}$} & 10 & 80 & 90 \\
\hline & \multirow{4}{*}{$B_{\text {méthode }}$} & bulles & 0 & 10 & 10 \\
\hline & & capillarité & 0 & 10 & 10 \\
\hline & & excès eau & 0 & 0 & 10 \\
\hline & & temps & 0 & négligeable & négligeable \\
\hline & \multirow{2}{*}{$B_{\text {moyens de mesure }}$} & zéro & 3 & 3 & 3 \\
\hline & & pesée & 3 & 3 & 3 \\
\hline & \multicolumn{2}{|c|}{$u_{m i}$} & 11 & 81 & 81 \\
\hline \multirow{8}{*}{$\mathrm{n}^{\circ} 3$} & \multicolumn{2}{|c|}{$\mathrm{A}$} & 10 & 130 & 130 \\
\hline & \multirow{4}{*}{$B_{\text {méthode }}$} & bulles & 0 & 10 & 10 \\
\hline & & capillarité & 0 & 10 & 10 \\
\hline & & excès eau & 0 & 0 & 10 \\
\hline & & temps & 0 & 50 & 50 \\
\hline & \multirow{2}{*}{$B_{\text {moyens de mesure }}$} & zéro & 3 & 3 & 3 \\
\hline & & pesée & 3 & 3 & 3 \\
\hline & \multicolumn{2}{|c|}{$u_{m i}$} & 11 & 205 & 205 \\
\hline
\end{tabular}

On admet que les erreurs associées à l'imbibition des échantillons entraînent une corrélation entre les masses $m_{2}$ et $m_{3}$. Dans un premier temps, on établit le budget des incertitudes évaluées par les méthodes de type $\mathrm{A}$ et de type $\mathrm{B}$ sur les trois pesées à partir de l'analyse présentée dans la section précédente. Puis, on calcule l'incertitude type composée pour chaque pesée $m_{i}$ selon l'expression suivante en admettant qu'il n'y a pas de corrélation entre les différentes composantes $u_{i}$ :

$$
u_{m_{i}}=\sqrt{\sum\left(u_{i}\right)^{2}} .
$$

Le tableau 6 donne les composantes d'incertitude sur les pesées pour les échantillons $\mathrm{n}^{\circ} 2$ et $\mathrm{n}^{\circ} 3$ dans le cas du protocole 2 . On admet que les composantes d'incertitude évaluées par une méthode de type B relatives au mesurande, à l'influence de l'opérateur et aux grandeurs d'influence sont négligeables par rapport aux autres composantes.

On constate que la composante de répétabilité (évaluée par une méthode de type A) est prépondérante par rapport aux autres composantes.

Pour déterminer l'incertitude sur la détermination de la porosité ouverte, il est nécessaire d'introduire les coefficients de sensibilité sur $m_{1}, m_{2}$ et $m_{3}$ qui ont pour expression :

$$
\begin{aligned}
& \frac{\partial \pi_{\mathrm{a}}}{\partial m_{1}}=-\frac{100}{m_{3}-m_{2}} \\
& \frac{\partial \pi_{\mathrm{a}}}{\partial m_{2}}=\frac{100 \times\left(m_{3}-m_{1}\right)}{\left(m_{3}-m_{2}\right)^{2}} \\
& \frac{\partial \pi_{\mathrm{a}}}{\partial m_{3}}=\frac{100 \times\left(m_{1}-m_{2}\right)}{\left(m_{3}-m_{2}\right)^{2}} .
\end{aligned}
$$

Le tableau 7 présente les valeurs de ces coefficients de sensibilité pour les échantillons $n^{\circ} 2$ et $n^{\circ} 3$ dans le cas du protocole 2 .

On détermine l'incertitude type composée sur $\pi_{\mathrm{a}}$ en tenant compte des corrélations en particulier entre $m_{2}$

\section{Tableau 7}

Masses mesurées et coefficients de sensibilité pour les échantillons $\mathrm{n}^{\circ} 2$ et $\mathrm{n}^{\circ} 3$ dans le cas du protocole 2 .

\begin{tabular}{|c|c|c|c|c|c|c|}
\hline Échantillon & $m_{1}(\mathrm{~g})$ & $m_{2}(\mathrm{~g})$ & $m_{3}(\mathrm{~g})$ & $\begin{array}{c}\frac{\partial \pi_{\mathrm{a}}}{\partial m_{1}} \\
\left(\mathrm{~g}^{-1}\right)\end{array}$ & $\begin{array}{c}\frac{\partial \pi_{\mathrm{a}}}{\partial m_{2}} \\
\left(\mathrm{~g}^{-1}\right)\end{array}$ & $\begin{array}{c}\frac{\partial \pi_{\mathrm{a}}}{\partial m_{3}} \\
\left(\mathrm{~g}^{-1}\right)\end{array}$ \\
\hline $\mathrm{n}^{\circ} 2$ & 151,44 & 94,61 & 181,13 & $-1,156$ & 0,397 & 0,759 \\
\hline $\mathrm{n}^{\circ} 3$ & 176,28 & $110,72^{*}$ & $187,69^{*}$ & $-1,299$ & 0,193 & 1,107 \\
\hline
\end{tabular}

* Valeurs corrigées de $2 \mathrm{~g}$ pour tenir compte de la masse d'eau manquante.

et $m_{3}$. Pour cela, on utilise l'expression générale suivante :

$$
u\left(\pi_{\mathrm{a}}\right)=\sqrt{\sum_{i=1}^{3} \sum_{j=1}^{3} \frac{\partial \pi_{\mathrm{a}}}{\partial m_{i}} \frac{\partial \pi_{\mathrm{a}}}{\partial m_{j}} r\left(m_{i}, m_{j}\right) u_{m i} u_{m j}}
$$

où $r\left(m_{i}, m_{j}\right)$ est le coefficient de corrélation entre les masses $m_{i}$ et $m_{j}$. Il vaut 1 pour $i=j$, on admet que la masse $m_{1}$ n'est pas corrélée avec les deux autres et que seuls les masses $m_{2}$ et $m_{3}$ sont corrélées entre elles. On prend un coefficient de +1 pour les composantes d'incertitude liées à la pénétration de bulles d'air et au temps d'imbibition. L'expression (19) peut s'écrire de la manière suivante :

$$
\begin{aligned}
& u\left(\pi_{\mathrm{a}}\right) \\
& =\sqrt{\sum_{i=1}^{3}\left(\frac{\partial \pi_{\mathrm{a}}}{\partial m_{i}}\right)^{2} u_{m i}^{2}+2 \times \frac{\partial \pi_{\mathrm{a}}}{\partial m_{2}} \frac{\partial \pi_{\mathrm{a}}}{\partial m_{3}}\left(u_{\text {air } 2} u_{\text {air } 3}+u_{\text {temps } 2} u_{\text {temps } 3}\right)} .
\end{aligned}
$$

En considérant le protocole 2 et en appliquant la correction de temps d'imbibition, on obtient les résultats suivants de porosité ouverte dont l'incertitude composée est exprimée avec un facteur d'élargissement $k=2$ :

- pour l'échantillon $n^{\circ} 2: \pi_{\mathrm{a}}=(34,32 \pm 0,14) \%$

- pour l'échantillon $n^{\circ} 3: \pi_{\mathrm{a}}=(14,82 \pm 0,46) \%$ 
On constate que la porosité ouverte est déterminée avec une incertitude composée élargie de l'ordre de $0,1 \%$ pour l'échantillon à large porosité et de $0,5 \%$ pour celui à fine porosité. Dans ce dernier cas, si le temps d'imbibition avait été porté à $3 \mathrm{~h}$ au lieu de $1 \mathrm{~h}$ selon le protocole 2, il n'y aurait pas eu besoin de faire de correction et la composante d'incertitude aurait pu être considérée comme négligeable. Seules les incertitudes types de répétabilité sur la détermination de $m_{2}$ et $m_{3}$ resteraient prépondérantes et conduiraient à une incertitude type composée de $0,13 \mathrm{~g}$ pour $m_{2}$ et $m_{3}$. Ainsi, on obtiendrait une incertitude composée élargie sur $\pi_{\mathrm{a}}$ de $0,3 \%$. Cependant, on peut raisonnablement penser qu'une meilleure saturation des pores après $3 \mathrm{~h}$ d'imbibition permettrait également d'améliorer significativement la répétabilité des pesées des masses $m_{2}$ et $m_{3}$ et par conséquent l'incertitude $\operatorname{sur} \pi_{\mathrm{a}}$.

\section{Conclusion}

Cette étude sur la détermination de la porosité ouverte de roches calcaires, utilisant les moyens de mesure disponibles au laboratoire selon une procédure s'inspirant d'une méthode normalisée, a permis de faire une analyse critique de la technique employée. La mise en œuvre d'une méthode « académique » d'analyse du processus de mesure (méthode des $5 \mathrm{M}$ ) a trouvé ici toute sa place et démontré son efficacité.

On peut regretter que les contraintes de temps aient conduit à limiter le nombre d'échantillons étudiés, le nombre de mesures effectuées ainsi que l'étude plus approfondie des conditions et grandeurs d'influence, ce qui n'est pas entièrement satisfaisant d'un point de vue métrologique. Néanmoins, cette étude a permis de démontrer que la méthode et le mode opératoire décrits dans la norme internationale NF ISO 5017 sont bien adaptés aux roches solides relativement denses et de structure homogène et qu'ils sont parfaitement justifiés et permettent d'éviter certains écueils qu'on a pu rencontrer dans la procédure utilisée.

Les principales sources d'erreur et de variabilité des résultats obtenus dans cette étude proviennent de :

La méthode de séchage/étuvage utilisée : on a amélioré la méthode de séchage/étuvage mise en œuvre en passant du protocole 1 au protocole 2 . On a pu constater le bien fondé de la recommandation spécifiée à ce sujet dans la Section 7.1 de la norme NF ISO 5017.

La phase d'imbibition: pour la phase d'imbibition, deux problèmes ont été mis en évidence : le dégazage de l'eau et le temps de saturation des pores. On a observé le dégazage, que ce soit avec de l'eau de ville, de l'eau désionisée ou de l'eau bidistillée, matérialisé par l'apparition d'une myriade de microbulles dans l'eau recueillie au fond du dessiccateur/saturateur à basse pression lorsque l'eau est injectée. Cependant, l'influence sur les pesées de l'échantillon imbibé n'a pas pu être mise en évidence. On a montré, par ailleurs, qu'il est impératif de respecter le temps nécessaire pour saturer les pores par l'eau. Un temps d'imbibition insuffisant peut entrâ̂ner un biais important sur la détermination de la porosité ouverte, tout particulièrement pour des matériaux à fine porosité.

La méthode d'essuyage appliquée : la méthode d'essuyage peut avoir une influence sur le résultat de pesée en termes de biais systématique et de répétabilité, car elle dépend beaucoup de l'opérateur. Cependant, les résultats obtenus n'ont pas mis en évidence de variabilité associée à l'essuyage.

La norme stipule que les valeurs de porosité doivent être données à $0,1 \%$ près en fraction volumique. On a montré que la méthode utilisée dans cette étude permet d'atteindre ce niveau d'incertitude pour la mesure de porosité ouverte pour des échantillons à large porosité. Il semble que cet objectif puisse être raisonnablement atteint pour des échantillons à fine porosité pour peu qu'on adapte le temps d'imbibition. Si l'on applique rigoureusement la procédure et les recommandations décrites dans la norme internationale, il semble tout à fait possible de déterminer la porosité ouverte à $0,1 \%$ près. De plus, si l'on connaît la masse volumique de la matrice avec une incertitude suffisamment faible, c'est-à-dire au moins du même ordre de grandeur que l'incertitude sur la mesure de porosité ouverte, on peut également déterminer les porosités fermée et totale avec cette même exactitude.

Cette méthode de mesure est bien adaptée aux matériaux solides à porosité homogène qu'ils soient réfractaires ou non. Pour son application à des roches naturelles, il est bien sûr essentiel de vérifier la représentativité des échantillons. Enfin, le niveau d'incertitude de mesure démontré et les grandeurs d'influence identifiées dans cette étude montrent qu'on peut adapter la capabilité de cette technique, selon les applications du matériau considéré et les exigences des utilisateurs, avec la mise en œuvre de moyens simples.

\section{Remerciements}

L'auteur remercie Simon Andrieu, étudiant en classes préparatoires au lycée Chaptal, pour avoir attiré son attention sur ce sujet d'étude.

\section{Références}

[1] Rouguerol J., AVNir D., FAIRBridge C.W., EVERETT D.H., HAYNeS J.H., PERnicone N., RAMSAY J.D.F., Sing K.S. W. et UNGER K.K., "Recommendations for the characterization of porous solids", Pure Appl. Chem., 66, 8, 1994, 1739-1758

[2] HARRISON D.J., "Industrial Minerals Laboratory Manual: Limestone”, BGS Technical report WG/92/29, Keyworth, Nottingham, British Geological Survey, 1993.

[3] Clarkson C.R., Jensen J.L. et Blasingame T.A., "Reservoir Engineering for Unconventional Gas Reservoirs: What Do We Have to Consider?", SPE North American Unconventional Gas Conference, The Woodlands, Texas, USA, June 2011, SPE 145080. 
[4] Solar C., García Blanco A., Vallone A. et Sapag K., "Adsorption of methane in porous materials as the basis for the storage of natural gas", Natural Gas, book edited by Primoz Potocnik, ISBN 978-953-307-112-1, Published: August 18, 2010, InTech, Available from: http://www.intechopen.com.

[5] Norme internationale : NF ISO 5017 « Produits réfractaires façonnés denses - Détermination de la masse volumique apparente, de la porosité ouverte et de la porosité totale », 3 Mai 2013, ISSN 0335-3931.

[6] HAYNES J.M., "Stereological analysis of pore structure", Matériaux et Constructions, 6, 33, 1973.

[7] LEMONS J.E. et RICHARDSON W.C., "Quantitative stereological investigations of porous alumina implant biomaterials, J. Dent. Res., 55, 1, 1976, 111-4.

[8] Biswal B., ØRen P.-E., Held R.J., BaKke S. et HiLfer R., "Modeling of multiscale porous media", Image Anal. Stereol., 28, 2009, 23-34.

[9] Mazumder S., SEn D. et PATRA A.K., "Characterization of porous materials by small-angle scattering", Pramana J. Phys., 63, 1, 2004, 165-173.

[10] WadE J.B., MARTin G.P. et LONG D.F., "An assessment of powder pycnometry as a means of determining granule porosity", Pharm. Dev. Technol. 2013, PMID: 24279883.

Article reçu le 3 juillet 2014, version révisée reçue le 12 février 2015.
[11] Chang C.S., "Measuring density and porosity of grain kernels using a gas pycnometer", Cereal Chem., 65, 1, 1998, 13-15.

[12] Zauer M., Pfriem A. et WagenfüHr A., "Toward improved understanding of the cell-wall density and porosity of wood determined by gas pycnometry", Wood Sci. Technol., 47, 2013, 1197-1211.

[13] Sweatman M.B. et QuiRKe N., "Characterization of Porous Materials by Gas Adsorption: Comparison of Nitrogen at $77 \mathrm{~K}$ and Carbon Dioxide at $298 \mathrm{~K}$ for Activated Carbon", Langmuir, 17, 2001, 5011-502.

[14] Feldblyum J.I., DutTa D., Wong-Foy A.G., DAILly A., IMIRZIAN J., GiDLEY D.W. et MATZGER A.J., "Interpenetration, Porosity, and High-Pressure Gas Adsorption in $\mathrm{Zn}_{4} \mathrm{O}$ (2,6-naphthalene dicarboxylate) $)_{3}$ ", Langmuir, 29, 25, 2013, 8146-8153.

[15] BARRETT E.P., JoYNER L.G. et HALENDA P.P., The determination of pore volume and area distributions in poroussubstances. I. Computations from nitrogen isotherms, $J$. Am. Chem. Soc., 73, 1951, 373-380.

[16] Abell A.B., Willis K.L. et Lange D.A., "Mercury Intrusion Porosimetry and Image Analysis of CementBased Materials", J. Coll. Interf. Sci., 211, 1, 1999, 39-44.

[17] BRUn M., LALlemand A., QUinson J.-F. et EyRAUd C., "A new method for the simultaneous determination of the size and shape of pores: the thermoporometry", Thermochim. Acta 21, 1, 1977, 59-88.

[18] Norme française : NF P 94-410-3 « Roches. Essais pour déterminer les propriétés physiques des roches. Partie 3 : Détermination de la porosité », mai 2001, ISSN 03353931. 